\title{
Lattice Simulations for Evaluating Interface Fracture of Masonry Composites
}

\author{
Amir Mohammadipour ${ }^{1}$, Kaspar Willam \\ Department of Civil and Environmental Engineering, University of Houston, \\ N110 Engineering Building 1, Houston, TX 77204-4003, USA
}

\section{Abstract}

The brick-mortar bond is often the weakest link in the masonry composites. The localization of fracture processes at this bi-material interface plays an important role in the failure of this assemblage. These micro-level fracture processes control the nonlinear behavior of the brick-mortar interface which significantly affects the global behavior of the masonry structure in the continuum macro level. This study focuses on 2-D lattice-based fracture simulations to characterize progressive debonding of brick-mortar interfaces and to determine fracture properties in unreinforced masonry composites. Using the fundamental argument of Griffith which transforms potential energy into surface energy, lattice erosion is used to determine the critical energy release rate and other fracture quantities from basic strength properties of lattice struts that are removed upon failure. This micro-level information serves to upscale the lattice fracture arguments onto the meso-scale to quantify the fracture energy of traction-separation cohesive zone models in the context of continuum F.E. simulations of heterogeneous media like masonry.

Keywords: Masonry composites; Lattice model; Interface fracture mechanics; Energy release rate; Stress intensity factor; Fracture toughness.

\section{INTRODUCTION}

Masonry is the oldest building material which is still used in building constructions around the world for its low cost material and broad availability, its sound insulation properties and energy efficiency, and also construction simplicity. There have been important new developments in analyzing masonry structures in the form of composite material in the last decades. However, due to the lack of in-depth insight and probably models for the complex behavior of masonry

\footnotetext{
${ }^{1}$ Corresponding author: Tel.: +18088619166.

E-mail address: amir_mohammadipour@yahoo.com, amohammadipour@uh.edu .
} 1 
composite consisting of brick, mortar, and the bond in between, its development of design rules has not kept pace with those of concrete and steel.

This might be one main reason to prevent the innovative applications of structural masonry. Unreinforced masonry is a heterogeneous, inelastic, and anisotropic material made of two major components, brick units and mortar joints exhibiting very different stiffness, strength and ductility properties. The brick-mortar interface which is the weakest part in the masonry composite plays an important role in the failure of this assemblage. A number of investigations have been conducted on different aspects of masonry and the interface behavior between brick and mortar joints, where an interface element was usually considered with a continuum-based damage or plasticity formulation to account for the brick-mortar interface degradation ([1], [2], [3], [4], [5], [6], [7], [8], [9], [10]). In the methods based on the theory of plasticity and damage mechanics, the displacement field is continuous over the domain and special techniques need to be accounted for embedding discontinuities and cracks on the domain. However, in the approaches based on fracture mechanics, the displacement field is discontinuous which accounts for the cracks and strong discontinuities.

There have been rare investigations regarding the interfacial fracture properties and toughness of masonry interfaces. In many bi-material systems like composites and microelectronic devices, the fracture of interfaces is a critical phenomenon, which in many circumstances governs the failure behavior of those systems. The fracture of bi-material interfaces has been studied by many researchers. Muskhelishvili [11] in his pioneering work employed the concept of complex variables and complex functions to represent the displacement and stress fields of plane problems using complex variables. He used complex functions since the properties of a complex variable are generally well-known. Williams [12] investigated the plane problem of dissimilar materials with a semi-infinite crack. He observed for the first time that stresses at the crack tip have an oscillatory character of type $r^{-1 / 2} \sin (\varepsilon \log r)$, where $r$ is the radial distance from the crack tip and $\varepsilon$ is a function of bi-material elastic mismatch. Rice and Sih [13] developed a method for determining Goursat functions for dissimilar materials bonded along straight-line interfaces. They combined an Eigen-function expansion method with the complex function of Muskhelishvili to solve the problems of isolated forces on surface of a semi-infinite crack and an infinite plate with a crack subjected to stresses at infinity. England [14], Erdogan [15], and Rice [16] also investigated the singular near-tip field of the interface crack problems. Parks [17] 
developed the "virtual crack extension" method which is a Finite Element technique for determining elastic crack tip stress intensity factors. In this method, the single crack is "advanced" by moving nodal points rather than by removing nodal tractions at the crack tip and performing a second analysis. Charalambides and his colleagues [18] devised an interesting test specimen which is capable of measuring the fracture resistance of bi-material interfaces. The test specimen is a four-point bending beam made of two dissimilar materials with a notch at the middle of the beam, as shown in Fig. 1. In their numerical Finite Element solutions, they obtained graphs for the energy release rate, stress intensity factor, and loading phase angle considering a pre-cracked notched symmetric composite beam model. Matos et al. [19] presented a numerical method for calculating stress intensity factors in bi-material interfaces. Their method is based on the $J$-integral using the "virtual crack extension" method, or energy method developed by Parks [17][20]. They compared the stress intensities obtained by the energy method and the "crack surface displacement" method. Charalambides et al. and Matos et al., in their simulations, considered a pre-cracked Finite Element mesh with length $a$ and applied the virtual crack extension method by virtually increasing the crack length and changing the stiffness of a ring of elements around the crack tip [17]. Their simulation was not based on a progressive crack propagation along the interface where the crack length $a$ increases during a single simulation. Evans et al. studied the fracture energy of bi-material interfaces and relative toughness of some bi-material interfaces with respect to the phase angle of loading [21]. Hutchinson and Suo [22], in their comprehensive paper, reviewed the investigations on fracture of layered materials including bi-material interfacial fracture mechanics.

\section{(Figure 1 is embedded here)}

Fig. 1 The pre-notched bi-material four-point bending beam with two symmetrical interfacial cracks [18]

\section{The Mechanics of InTERfaCe Cracks}

Consider two isotropic elastic solids which are in contact through their interface along $x$-axis as shown in Fig. 2. Material 1 is above the interface and the origin is at the crack tip. Let $\mu_{j}, E_{j}$, and $v_{j}(j=1,2$ as material index) be the shear modulus, Young's modulus, and Poisson's ratio 
of two materials. Dundur's elastic mismatch parameters [23] can be employed for a wide class of plane problems of elasticity.

\section{(Figure 2 is embedded here)}

Fig. 2 A small region near crack tip along bi-material interface.

Since there is displacement continuity along the interface, it follows that

$$
\left(\varepsilon_{x}\right)_{1}=\left(\varepsilon_{x}\right)_{2} \text { along interface, }
$$

where $(.)_{1}$ and $(.)_{2}$ belong to material 1 and 2, respectively. From Eq. (1) and for plane strain

$$
\left(\sigma_{x}\right)_{1}=\frac{\mu_{1}\left(1-v_{2}\right)}{\mu_{2}\left(1-v_{1}\right)}\left(\sigma_{x}\right)_{2}+\frac{\sigma_{y}}{1-v_{1}}\left(v_{1}-\frac{\mu_{1}}{\mu_{2}} v_{2}\right) .
$$

If a parameter called $\alpha$ is defined as below,

$$
\frac{\mu_{1}\left(1-v_{2}\right)}{\mu_{2}\left(1-v_{1}\right)}=\frac{1+\alpha}{1-\alpha}
$$

Then the Dundur's parameter $\alpha$ for plane strain can be expressed in terms of the elastic properties of the adherent linear isotropic media

$$
\alpha=\frac{\mu_{1}\left(1-v_{2}\right)-\mu_{2}\left(1-v_{1}\right)}{\mu_{1}\left(1-v_{2}\right)+\mu_{2}\left(1-v_{1}\right)} .
$$

Thus, Eq. (2) can be represented as

$$
\left(\sigma_{x}\right)_{1}=\frac{1+\alpha}{1-\alpha}\left(\sigma_{x}\right)_{2}+\frac{2 \sigma_{y}}{1-\alpha}(2 \beta-\alpha)
$$

where $\alpha$ and $\beta$ are two Dundur's elastic mismatch parameters which are generally expressed by

$$
\begin{gathered}
\alpha=\frac{\mu_{1}\left(\kappa_{2}+1\right)-\mu_{2}\left(\kappa_{1}+1\right)}{\mu_{1}\left(\kappa_{2}+1\right)+\mu_{2}\left(\kappa_{1}+1\right)}, \text { and } \\
\beta=\frac{\mu_{1}\left(\kappa_{2}-1\right)-\mu_{2}\left(\kappa_{1}-1\right)}{\mu_{1}\left(\kappa_{2}+1\right)+\mu_{2}\left(\kappa_{1}+1\right)},
\end{gathered}
$$

where $\kappa_{j}=3-4 v_{j}$ for plane strain and $\kappa_{j}=\left(3-v_{j}\right) /\left(1+v_{j}\right)$ for plane stress. The $\alpha$ parameter is a measure of the mismatch in the in-plane tensile modulus across the interface, while $\beta$ measures the mismatch in the in-plane bulk moduli [22]. Crack tip displacement and stress fields oscillate when $\beta \neq 0$, which results in crack surface interpenetration. This interpenetration introduces ambiguity into the characteristics of interface fracture [16,21]. 
However, since for many bi-material systems of interest including masonry and concrete [24], the value of $\beta$ is small [25], it is assumed that a zero- $\beta$ hypothesis should provide an adequate interface fracture characterization in many cases.

Muskhelishvili [11] showed that any problem in the plane theory of elasticity can be solved by finding two complex functions, satisfying the boundary conditions of that problem. However, in bi-material systems, the elastic properties are discontinuous across the interface, where four complex functions, Goursat functions, $\Phi_{j}(z), \Psi_{j}(z), j=1,2$, of the complex variable $z=x+$ $i y$ are needed to completely characterize the problem $(i=\sqrt{-1})$. The basic equations for displacement and stress fields for two dimensional isotropic elasticity as used by KolosovMuskhelishvili are [13]

$$
\begin{gathered}
\left(\sigma_{x}\right)_{j}+\left(\sigma_{y}\right)_{j}=4 \operatorname{Re}\left[\Phi_{j}(z)\right], \\
\left(\sigma_{y}\right)_{j}-\left(\sigma_{x}\right)_{j}+2 i\left(\tau_{x y}\right)_{j}=2\left[\bar{z} \Phi_{j}^{\prime}(z)+\Psi_{j}(z)\right],
\end{gathered}
$$

and

$$
2 \mu_{j}\left(u_{j}+i v_{j}\right)=\kappa_{j} \int \Phi_{j}(z) d z-z \overline{\Phi_{j}}(\bar{z})-\int \overline{\Psi_{j}}(\bar{z}) d \bar{z},
$$

where $u_{j}, v_{j}$ are components of displacement along $x$ and $y$ axes, shown in Fig. 2, for material $j$, $\left(\sigma_{x}\right)_{j},\left(\sigma_{y}\right)_{j},\left(\tau_{x y}\right)_{j}$ are components of stress, $\mu_{j}$ denotes the shear moduli, and $\kappa_{j}$ represents the Poisson's ratios.

Using Muskhelishvili's Eqs. (7), (8), (9) mentioned above and the development of the singular near-tip field of the interface crack problem (Fig. 2) by [14], [15], and [13], the tractions on the interface directly ahead of the tip $(\theta=0)$ are given by

$$
\left(\sigma_{y}\right)_{j}+i\left(\tau_{x y}\right)_{j}=K(2 \pi r)^{-1 / 2} r^{i \varepsilon},
$$

or

$$
\left(\sigma_{y}\right)_{j}=\operatorname{Re}\left[K r^{i \varepsilon}\right](2 \pi r)^{-1 / 2},\left(\tau_{x y}\right)_{j}=\operatorname{Im}\left[K r^{i \varepsilon}\right](2 \pi r)^{-1 / 2},
$$

where $K=K_{1}+i K_{2}$ is the complex stress intensity factor, $r^{i \varepsilon}=e^{i \varepsilon \ln (r)}=\cos (\varepsilon \ln (r))+$ $i \sin (\varepsilon \ln (r))$ is a so-called oscillatory singularity, and $\varepsilon$ is the bi-elastic constant

$$
\varepsilon=\frac{1}{2 \pi} \ln \left(\frac{1-\beta}{1+\beta}\right) .
$$


The relative crack flank displacements of two points on the top and bottom of crack surfaces, $\Delta u$ and $\Delta v$, at a distance $r$ behind the crack tip are given by

$$
\Delta v+i \Delta u=\frac{8 K \sqrt{\frac{r}{2 \pi}}}{(1+2 i \varepsilon) \cosh (\pi \varepsilon) E_{*}} r^{i \varepsilon},
$$

where $\Delta u=u(r, \theta=\pi)-u(r, \theta=-\pi), \Delta v=v(r, \theta=\pi)-v(r, \theta=-\pi), \frac{1}{E_{*}}=\frac{1}{2}\left(\frac{1}{\bar{E}_{1}}+\frac{1}{\bar{E}_{2}}\right)$ and $\bar{E}_{j}=E_{j} /\left(1-v_{j}^{2}\right)$ for plane strain and $\bar{E}_{j}=E_{j}$ for plane stress. For plane strain problems, Eq. (12) reduces to

$$
\Delta v+i \Delta u=\frac{2\left[\frac{1-v_{1}}{\mu_{1}}+\frac{1-v_{2}}{\mu_{2}}\right] K \sqrt{\frac{r}{2 \pi}}}{(1+2 i \varepsilon) \cosh [(\pi \varepsilon)} r^{i \varepsilon}
$$

The complex stress intensity factor $K$ can generally be expressed in terms of its modulus $|K|=$ $\sqrt{{K_{1}}^{2}+K_{2}{ }^{2}}$, and the loading phase angle $\psi$ by

$$
K=|K| e^{i \psi}
$$

Since the crack surface displacement is a complex number, $\Delta v+i \Delta u$, it can also be written as $\Delta v+i \Delta u=\left[\Delta u^{2}+\Delta v^{2}\right]^{1 / 2} e^{i \phi}$, where $\phi=\arctan \left(\frac{\Delta u}{\Delta v}\right)$. By equating this with Eq. (13) and after some manipulations, $|K|$ can be expressed in terms of the crack surface displacements as [18]

$$
|K|=\left[\left(1+4 \varepsilon^{2}\right)\left(\Delta u^{2}+\Delta v^{2}\right)\right]^{1 / 2} / q
$$

where $q=\sqrt{2}\left[\frac{1-v_{1}}{\mu_{1}}+\frac{1-v_{2}}{\mu_{2}}\right] /(\sqrt{\pi} \cosh (\pi \varepsilon))$. The loading phase angle is given by

$$
\psi=\omega+\gamma,
$$

where $\gamma=\arctan (2 \varepsilon)$ is the principal argument of the complex number $1+i 2 \varepsilon$, and $\omega=\phi-$ $\varepsilon \ln (r)$. Moreover, the energy release rate and the modulus of stress intensity are correlated by the Irwin-type relation below

$$
G=\frac{\left(1-\beta^{2}\right)|K|^{2}}{E_{*}}=\frac{|K|^{2}}{E_{*} \cosh ^{2}(\pi \varepsilon)} .
$$

For plane strain problem, $|K|$ is related to $G$ by 


$$
|K|=\sqrt{\frac{4 \cosh ^{2}(\pi \varepsilon) G}{\frac{1-v_{1}}{\mu_{1}}+\frac{1-v_{2}}{\mu_{2}}}} .
$$

Eqs. (15) to (18) can be used to obtain interfacial fracture quantities from numerical solutions. The energy release rate, $G$, can firstly be calculated through an energy method like virtual crack extension procedure [17]. Other numerical solutions such as lattice model can also be employed to obtain $G$. This will be explained in detail further in this article. Eq. (18) can then directly be used to obtain $|K|$, which is independent of the crack flank displacements and the distance $r$ behind the crack tip. As an internal consistency check, the relative near tip crack surface displacements may be inserted into Eq. (15) to obtain an independent estimate of $|K|$ to be compared with that of Eq. (18) [19]. The stress intensity factors for mode 1 and mode 2 are then calculated using the phase angle by

$$
\begin{gathered}
K_{1}=\operatorname{Re}(K)=|K| \cos ((\psi), \text { and } \\
K_{2}=\operatorname{Im}(K)=|K| \sin ((\psi) .
\end{gathered}
$$

In circumstances when the Dundur's parameter $\beta$ is nonzero and thus $\varepsilon \neq 0$, a pure mode 1 crack with zero shear traction along the interface happens at a distance $\hat{L}$ ahead of the tip and a pure mode 2 crack with zero normal traction on the interface is at that point. Since the ratio of the shear traction to normal traction on the interface varies (very slowly) with distance to the tip when $\beta \neq 0$, the measure of the proportion of mode 2 to mode 1 in the vicinity of the crack tip requires the specification of some length quantity, $\widehat{L}$ [22]. The choice of this characteristic length, $\widehat{L}$, is somewhat arbitrary but when chosen it should not change throughout the analysis. This characteristic length establishes a new rotated quantity $\widehat{K}_{1}+i \widehat{K}_{2}$ with the same modulus $|K|$ as $K_{1}+i K_{2}$ (Note that $\left|\hat{L}^{i \varepsilon}\right|=1$ and $\left|K \hat{L}^{i \varepsilon}\right|=|K|$ ) but an augmented phase angle $\hat{\psi}$ which unlike $\psi$ is insensitive to the choice of length unit [19]. Thus,

$$
\begin{gathered}
K=|K| e^{i \psi} \stackrel{\times \hat{L}^{i \varepsilon}}{\Longrightarrow} \\
K \hat{L}^{i \varepsilon}=|K| e^{i \psi} \hat{L}^{i \varepsilon} \stackrel{\hat{L}^{i \varepsilon}=e^{i \varepsilon \ln \widehat{L}}}{=} K \hat{L}^{i \varepsilon}=|K| e^{i \psi} e^{i \varepsilon \ln \hat{L}}=|K| e^{i(\psi+\varepsilon \ln \hat{L})}=|K| e^{i \widehat{\psi}} \Rightarrow \\
K \hat{L}^{i \varepsilon}=|K| e^{i \widehat{\psi}},
\end{gathered}
$$


where

$$
\widehat{\psi}=\psi+\varepsilon \ln \hat{L} .
$$

From Eq. (16),

$$
\begin{gathered}
\psi=\omega+\gamma=\phi-\varepsilon \ln (r)+\gamma \stackrel{+\varepsilon \ln \hat{L}}{=} \psi+\varepsilon \ln \hat{L}=\phi-(\varepsilon \ln (r)-\varepsilon \ln \hat{L})+\gamma \\
=\phi-\varepsilon \ln \left(\frac{r}{\widehat{L}}\right)+\gamma \Rightarrow \\
\qquad \hat{\psi}=\phi-\varepsilon \ln \left(\frac{r}{\widehat{L}}\right)+\gamma .
\end{gathered}
$$

Therefore, the value of $\psi$ is replaced by $\hat{\psi}$ in Eqs. (16), (19), and (20) when the characteristic specimen length $\hat{L}$ is introduced in the analysis and the modulus of $K$ remains the same. In this study, $\hat{L}$ is usually considered as specimen height, e.g. $h_{1}+h_{2}$ in Fig. 1.

\section{NUMERICAL IMPLEMENTATION: LATTICE MODEL}

Heterogeneous materials exhibit complicated fracture mechanisms due to their microstructure. Analytical descriptions of these mechanisms using linear elastic fracture mechanics is difficult, since the fracture pattern includes a main crack, itself consists of various branches, secondary cracks, and micro-cracks. Due to the limitations and inflexible nature of analytical methods in handling arbitrary complex geometries and boundary conditions and general crack propagations, fracture processes in heterogeneous materials are, therefore, often simulated with numerical models. One of the numerical tools which is well suited for fracture simulations is the lattice model. The main concept was first introduced by Hrennikoff [26], where a continuum domain was discretized in a lattice of truss or beam or frame elements.

\section{(Figure 3 is embedded here)}

Fig. 3 Discretization process of a rectangular continuum domain into particles and lattice struts using Voronoi formulation, (a) a regular discretization, (b) a random discretization, (c) a regular lattice, (d) a random lattice.

Voronoi diagrams, based on a random or regular distribution of points, were used here to discretize the continuum domain into an assemblage of convex rigid particles interconnected 
along their boundaries through flexible common sides or interfaces [27] (Fig. 3). This formulation was used to generate lattice meshes in this study as illustrated in Fig. 3. This diagram is uniquely defined by the nuclei distribution. The Voronoi region, or cell, assigned to nucleus $i$ is [27] (Fig. 4a)

$$
V_{i} \stackrel{\text { def }}{=} V\left(\boldsymbol{X}_{\boldsymbol{i}}\right)=\bigcap_{j \neq i}\left\{\boldsymbol{X} \mid d\left(\boldsymbol{X}_{\boldsymbol{i}}, \boldsymbol{X}\right) \leq d\left(\boldsymbol{X}_{\boldsymbol{j}}, \boldsymbol{X}\right)\right\},
$$

\section{(Figure 4 is embedded here)}

Fig. 4 (a) Voronoi particle associated with nucleus i. Line segment i-j establishes a frame element, (b) embedding translational and rotational stiffness between two particles on the interface, (c) facet local displacement in $t-n$ coordinates [27].

where $\boldsymbol{X}_{\boldsymbol{i}}$ are the coordinates of nucleus $i ; d\left(\boldsymbol{X}_{\boldsymbol{i}}, \boldsymbol{X}\right)$ is the Euclidean distance between $\boldsymbol{X}_{\boldsymbol{i}}$ and $\boldsymbol{X}$; and $j$ runs from 1 to $n$, excluding $i$. Similarly, the boundary, or interface, segment common to two contiguous particles $i$ and $j$ is [27] (Fig. 4a)

$$
S_{i j} \stackrel{\text { def }}{=} S\left(\boldsymbol{X}_{\boldsymbol{i}}, \boldsymbol{X}_{\boldsymbol{j}}\right)=\cap_{k \neq i, j}\left\{\boldsymbol{X} \mid d\left(\boldsymbol{X}_{\boldsymbol{i}}, \boldsymbol{X}\right)=d\left(\boldsymbol{X}_{\boldsymbol{j}}, \boldsymbol{X}\right) \leq d\left(\boldsymbol{X}_{\boldsymbol{k}}, \boldsymbol{X}\right)\right\} .
$$

Although Eqs. (24) and (25) are conceptually simple, the generation of Voronoi diagram is nontrivial due to computing demands and other issues such as the modeling of domain boundaries. Thus, special care was exercised to account for domain boundaries, both in regular and random meshes. Moreover, 2-D 'frame' elements with three degrees of freedom at nodes (nuclei) were considered in this study. Schlangen and Garboczi [28] evaluated three types of elements, i.e. trusses with 1,2, and frames with 3 degrees of freedom at each node. They concluded that in the simulations with frame elements, the crack pattern on the mesh was much closer to the experimentally obtained cracks. J. van Mier [29] also recommended frame elements as they can be fitted over a wider range to match the elastic constants of uncracked concrete.

\section{(Figure 5 is embedded here)}

Fig. 5 Overlaying material properties onto lattice structure. Black, blue and pink represent brick, mortar, and interface material properties, respectively, (a) coarser mesh, (b) finer mesh. 
One of the attractions of lattice models is the combination of the mechanics model and the material structure, called material structure overlay. The lattice is the mechanical model; the material structure is simply projected on top of the lattice and various properties are assigned to the lattice elements depending on their specific location in the projectled material structure. Fig. 5 illustrates the material properties' assignment to the mechanical model.

With regard to mechanical relationships between two particles (Figs. 4b, $4 \mathbf{c}$ ) two triangular particles are connected at their interfaces by translational and rotational stiffnesses, i.e. $k_{n}, k_{t}$, and $k_{\varphi}$, which approximate the elastic properties of the continuum. The relation between internal forces and local displacements is [27]

$$
q=D d
$$

where $\boldsymbol{q}^{\boldsymbol{t}}=\left\{q_{n}, q_{t}, q_{\phi}\right\}$ is the internal force vector between two particles; $\boldsymbol{D}$ is a diagonal matrix containing the normal, tangential, and rotational spring stiffnesses depicted in Fig. $\mathbf{4 b}$, i.e. $\boldsymbol{D}=\operatorname{diag}\left[k_{n}, k_{t}, k_{\phi}\right]$; and $\boldsymbol{d}^{t}=\left\{\delta_{n}, \delta_{t}, \phi\right\}$ is the local relative displacement vector of two particles in the normal, tangential, and rotational directions (Fig. 4c).

The spring stiffnesses are obtained in terms of elastic properties of the continuum domain and also the geometry of the connected particles. For brick and mortar frame elements

$$
\begin{gathered}
k_{n}=\frac{E^{\prime} A}{h_{0}}=\frac{E^{\prime} t l_{43}}{h_{0}}, \\
k_{t}=\frac{E^{\prime \prime} A}{h_{0}}=\frac{E^{\prime \prime} t l_{43}}{h_{0}}, \text { and } \\
k_{\phi}=\frac{E^{\prime} I}{h_{0}}=\frac{E^{\prime}}{h_{0}}\left(\frac{t l_{43}{ }^{3}}{12}\right)=\frac{E^{\prime}\left(t \times l_{43}\right)}{h_{0}}\left(\frac{l_{43}{ }^{2}}{12}\right)=\frac{E^{\prime}(A)}{h_{0}}\left(\frac{l_{43}{ }^{2}}{12}\right)=\frac{k_{n} l_{43}{ }^{2}}{12},
\end{gathered}
$$

where $E^{\prime}=E /\left(1-v^{2}\right)$ and $E^{\prime \prime}=\mu=E /[2(1+v)]$ for plane stress and $E^{\prime}=E(1-v) /[(1+v)(1-2 v)]$ and $E^{\prime \prime}=\mu=E /[2(1+v)]$ for plane strain; $E$ and $v$ are the Young's modulus and Poisson ratio of the continuum material, respectively; $t$ is the specimen thickness; $h_{0}$ is the frame element length between points 1 and 2 , as shown in Fig. $\mathbf{4 b}$; $l_{43}$ is the frame element width between points 3 and 4 in Fig. $\mathbf{4 b}$; and $A=t l_{43}$ is the cross sectional area. For the interface elements, $k_{n}=K_{n} A, k_{t}=K_{t} A$, and $k_{\phi}=0$, similar to truss elements with no rotational stiffnesses. $K_{n}$ and $K_{t}$ are obtained by homogenizing the composite material surrounding the interface, i.e. brick and mortar [10] 


$$
\begin{gathered}
K_{n}=\frac{E_{b} E_{m}}{h_{b} h_{m}\left(E_{b} / h_{b}+{ }^{E_{m} / h_{m}}\right)}, \text { and } \\
K_{t}=\frac{\mu_{b} \mu_{m}}{h_{b} h_{m}\left({ }^{\mu_{b}} / h_{b}{ }^{\mu_{m} / h_{m}}\right)},
\end{gathered}
$$

where $E_{b}, \mu_{b}$ and $E_{m}, \mu_{m}$ are the normal and shear moduli of the brick and mortar, respectively. Eqs. (28) and (29) can be obtained by considering a serial arrangement of brick-interface-mortar where the volume change is negligible.

The stiffness matrix in global $x-y$ coordinates associated with the two-particle assemblage follows from matrix analysis of structures

$$
\boldsymbol{k}_{e}=B^{t} \mathrm{DB}
$$

where the matrix $\boldsymbol{B}$ relates the displacement vector in local $t-n$ coordinates, i.e. $\boldsymbol{d}$, to that in global $x-y$ coordinates, i.e. $\boldsymbol{u}_{\boldsymbol{e}}{ }^{t}=\left\{u_{1}, v_{1}, \theta_{1}, u_{2}, v_{2}, \theta_{2}\right\}$ as [27]

$$
\begin{gathered}
\boldsymbol{d}=\boldsymbol{B} \boldsymbol{u}_{\boldsymbol{e}}= \\
\frac{1}{l_{43}}\left[\begin{array}{cccccc}
y_{43} & -x_{43} & \left(-x_{43} x_{P 1}-y_{43} y_{P 1}\right) & -y_{43} & x_{43} & \left(x_{43} x_{P 2}+y_{43} y_{P 2}\right) \\
x_{43} & y_{43} & \left(y_{43} x_{P 1}-x_{43} y_{P 1}\right) & -x_{43} & -y_{43} & \left(-y_{43} x_{P 2}+x_{43} y_{P 2}\right) \\
0 & 0 & -l_{43} & 0 & 0 & l_{43}
\end{array}\right]\left\{\begin{array}{l}
u_{1} \\
v_{1} \\
\theta_{1} \\
u_{2} \\
v_{2} \\
\theta_{2}
\end{array}\right\},
\end{gathered}
$$

where point $P$ is the facet midpoint in Fig. $\mathbf{4 b}$ and $x_{i j}=x_{i}-x_{j}, y_{i j}=y_{i}-y_{j}$. Therefore,

$$
\left\{\begin{array}{l}
x_{P i}=\left(x_{4 i}+x_{3 i}\right) / 2 \\
y_{P i}=\left(y_{4 i}+y_{3 i}\right) / 2
\end{array} \quad i=1,2 .\right.
$$

Also,

$$
\boldsymbol{k}_{e} \boldsymbol{u}_{e}=\boldsymbol{f}_{\boldsymbol{e}}
$$

in which $\boldsymbol{f}_{\boldsymbol{e}}$ contains the force components in global coordinates for the frame element associated with each respective entry in displacement vector $\boldsymbol{u}_{\boldsymbol{e}}$.

The simulation of fracture, in this study, was performed with a 'linear elastic' analysis of the lattice under loading and removing an element from the mesh which exceeds a certain fracture criterion, for instance a tensile or compressive stress based on the failure envelope. The 'gap' between the remaining elements is considered as a discontinuity or crack in the lattice mesh. After removing the element, the lattice mesh contains one less element. The simulation is continued by performing a linear elastic analysis of the new mesh, where the forces that were 
carried by the removed element are now redistributed over the neighboring elements. This procedure continues until the next element satisfies its 'fracture criterion', and so on [28], [29]. Thus, in each step, the external load on the lattice is increased and the critical element at the fracture threshold is removed. The erosion strategy leads to an 'instantaneous relaxation' of the load, carried by that removed part of the lattice [29]. This was often observed during the lattice analyses of this study as a sudden drop in form of snap-backs in the load-displacement diagrams. Fracture criterion for the failure of brick and mortar frame elements, i.e. "black" and "blue" struts in Fig. 5, was defined as a function of normal force and bending moments at computational points of each frame member

$$
\sigma_{e f f}=\frac{N}{A} \pm \alpha^{\prime} \frac{\left(\left|M_{i}\right|,\left|M_{j}\right|\right)_{\max }}{S} \geq f_{t} \text { or } f_{c}
$$

where $N$ is the normal force of the lattice element; $A$ is its cross sectional area; $M_{i}$ and $M_{j}$ are the bending moments at the nodes $i$ and $j$, respectively; $S=l_{43} t^{2} / 6$ is the section modulus; $f_{t}$ and $f_{c}$ are the tensile and compressive strengths of the material, respectively; and $0 \leq \alpha^{\prime} \leq 1$ is added to limit the effect of bending in the fracture law. The value of $\alpha^{\prime}$ was determined from parametric studies and comparison with experimental measurements [30].

\section{(Figure 6 is embedded here)}

Fig. 6 Failure surface for the brick-mortar interface employed in this study.

The fracture criterion for the brick-mortar interface was determined in this study based on a combination of experimental measurements and numerical parameter simulations. Fig. 6 shows the fracture condition considered for the interface frame elements in the lattice model. This failure envelope has a compressive cap which was necessary for the simulation of triplet test under normal confinement. In this figure, the failure surface has three major parts, namely a tension cut-off, a Coulomb shear envelope, and a compressive cap. The tension cut-off part, $f_{t}$, was determined based on the direct tension test of interface [31] while the inclined shear envelope, $f_{s}$ and $\mu_{0}$, was obtained by the triplet experiments as explained in [31]. The horizontal part of the shear envelope in Fig. 6 and also the compressive cap, $f_{c}$, were determined by parametric studies of confined triplet simulations under different high confinements in the implemented lattice model. It should be noted that the shear failure surfaces were neglected for 
brick and mortar elements since the main focus of this study was to evaluate the brick-mortar interface fracture properties, and with the simulations conducted, their shear failure envelopes were not activated. Table 1 presents the material properties of the brick, mortar, inteface elements needed for the lattice analysis determined form both experimental programs and numerical parametric studies.

The material properties of the interface explained above in Fig. 6 were put in Table $1 . K_{n}$ and $K_{t}$ of the interface were determined according to Eqs. (28) and (29) explained earlier. Material properties of brick and mortar in Table 1 were measured by conducting compression tests of brick units and mortar prisms to obtain Young's moduli, $E$, and compressive strengths, $f_{c}$, of the specimens. The tensile strengths were measured by performing splitting tension or Brazilian tests which is an indirect tension test $[32,33]$. The splitting tension test complies with the specifications of ASTM C 1006-84 [34]. In order to measure specimens' deformation to account for $E$ and $v$, a Digital Image Correlation (DIC) system which is a non-contact optical 3D metrology system was used in all experiments. In the DIC technique, the software processes the images taken during the test to determine the full-field motion of the speckle geometry, and obtains surface deformations in terms of strain measurements. Readers are kindly referred to [32] for more details. Because of the brittle behavior of the specimens, there were difficulties to measure the lateral deformation of the specimens using the DIC to obtain Poisson's ratios. The values reported in Table 1 were determined based on the measurements and those reported in literature for brick and mortar.

\section{(Table 1 is embedded here)}

Table 1 Mechanical characteristics of materials adopted for the computations

\section{SimULATIONS AND RESULTS}

The concepts of interfacial fracture mechanics explained earlier were used in this study along with the implementation of the lattice model to determine the fracture properties of the brickmortar interface. All the simulations in this study were implemented in MATLAB R2014a. The approach can be used for any other bi-material interfaces. Charalambides et al. [18], Matos et al. [19], and others used a numerical F.E. approach where a 'pre-cracked' Finite Element mesh with 
a fixed length $a$ was considered and the virtual crack extension method was applied by virtually increasing the crack length and changing the stiffness of a ring of elements around the crack tip. In the implemented lattice model, the strut removal strategy is used and the discontinuity or rather the crack development in the computation of domain is recorded. Therefore unlike the 'pre-cracked' approach, this lattice model is capable of capturing the fracture properties of an interface based on progressive crack propagation along the interface where the crack length $a$ increases during a single simulation.

As mentioned before, Eqs. (15) to (18) can be used to obtain interfacial fracture quantities from numerical solutions. The main quantity to be calculated is the energy release rate of the interface fracture. This value is determined by an energy approach using the total potential energy, $\Pi$, of the lattice solution. Assume that a lattice analysis has been performed on a given planar linear elastic body of 'unit thickness' containing a crack. The total potential energy of the lattice model solution may be expressed as [35], [17]

$$
\Pi=\frac{1}{2}\{u\}^{T}[K]\{u\}-\{u\}^{T}\{f\},
$$

where $\{u\}$ is a vector of displacements associated with lattice computational (nodal) points or nuclei, $[K]$ is the global stiffness matrix of the lattice mesh, and $\{f\}$ is the vector of prescribed nodal loads. The energy release rate is obtained by differentiating Eq. (34) with respect to crack length, $a$, as [36]

$G=-\frac{\partial \Pi}{\partial a}=-\frac{\partial}{\partial a}\left[\{u\}^{T}\left(\frac{1}{2}[K]\{u\}-\{f\}\right)\right]=-\left[\frac{\partial\{u\}^{T}}{\partial a}\left(\frac{1}{2}[K]\{u\}-\{f\}\right)+\{u\}^{T}\left(\frac{1}{2} \frac{\partial[K]}{\partial a}\{u\}+\right.\right.$ $12 K \partial u \partial a-\partial f \partial a$

$G=-\left[\frac{\partial\{u\}^{T}}{\partial a}\left(([K]\{u\}-\{f\})-\frac{1}{2}[K]\{u\}\right)+\left(\frac{1}{2}\{u\}^{T} \frac{\partial[K]}{\partial a}\{u\}+\frac{1}{2}\{u\}^{T}[K] \frac{\partial\{u\}}{\partial a}-\{u\}^{T} \frac{\partial\{f\}}{\partial a}\right)\right]$.

The value of $([K]\{u\}-\{f\})$ is precisely zero in the finite element and lattice equilibrium solutions at each iteration. Thus, $G=-\left[\left(-\frac{1}{2} \frac{\partial\{u\}^{T}}{\partial a}[K]\{u\}+\frac{1}{2}\{u\}^{T}[K] \frac{\partial\{u\}}{\partial a}\right)+\left(\frac{1}{2}\{u\}^{T} \frac{\partial[K]}{\partial a}\{u\}-\{u\}^{T} \frac{\partial\{f\}}{\partial a}\right)\right]$.

Since the stiffness matrix $[K]$ is symmetric, it can easily be shown that the scalar value on the first parenthesis is zero. This follows that

$$
G=-\left[\frac{1}{2}\{u\}^{T} \frac{\partial[K]}{\partial a}\{u\}-\{u\}^{T} \frac{\partial\{f\}}{\partial a}\right]
$$


Since the crack surfaces here are traction free, one can conclude that $\frac{\partial\{f\}}{\partial a}=0$. Hence,

$$
G=-\frac{\partial \Pi}{\partial a}=-\frac{1}{2}\{u\}^{T} \frac{\partial[K]}{\partial a}\{u\}
$$

Eq. (36) is the main ingredient to obtain the fracture properties of the interface. $\frac{\partial[K]}{\partial a}$ is the change in the global stiffness matrix when the change in crack length is $\partial a$, and $\{u\}$ denotes the nuclei displacement vector before the crack length change by $\partial a$. In the numerical solution, $\frac{\partial[K]}{\partial a}$ is approximated by the ratio $\frac{\Delta[K]}{\Delta a}$ as

$$
\frac{\partial[K]}{\partial a} \cong \frac{\Delta[K]}{\Delta a}=\frac{1}{\Delta a}\left[[K]_{a+\Delta a}-[K]_{a}\right]
$$

where $[K]_{a+\Delta a}$ is the stiffness matrix after the crack growth $\Delta a$. Therefore, using Eq. (36) the interfacial energy release rate can numerically be determined by lattice analysis as the crack propagates through the interface. It should be mentioned that the value of $G$ obtained from Eq. (36) is for a specimen with unit thickness. If the thickness $t \neq 1$, then $G$ should be divided by $t$.

\subsection{Lattice Simulations of Some Classic Fracture Problems}

The implemented 2D lattice model is capable of simulating crack path evolution in the form of strong discontinuities at a homogeneous or heterogeneous solid. In this section, three wellknown classic crack problems the analytical solutions of which have been obtained are simulated by the implemented lattice model. The stress intensity factors calculated by the analytical solutions are compared with those obtained by the lattice to confirm its numerical capabilities in predicting the desired fracture quantities.

\subsubsection{The Center Cracked Lattice Simulation}

In order to validate the fracture analysis results of the lattice, it is required to compare its numerical results with the classic analytical solutions available in the literature. Tada, Paris and Irwin [37] presented a comprehensive review of stress analysis of cracks from two dimensional common test specimens to three dimensional cracked configurations. Fig. 7a illustrates the homogeneous finite width center cracked test specimen with constant thickness on which a far field tensile stress is applied. Mode 1 crack tip stress intensity factor of this problem is expressed as [37] 


$$
K_{1}=\sigma \sqrt{\pi a} F(a / b)
$$

where $2 a$ is the center crack length, $\sigma$ is the tensile far field stress, $2 b$ is the width of the plate or configuration as illustrated in Fig. 7a, and $F(a / b)$ is an empirical relation. In this study, $F(a / b)$ with $0.3 \%$ accuracy of any $a / b$ which was obtained with a modification of Koiter's formula was considered as defined by [37], [38]

$$
F(a / b)=\left\{1-0.025(a / b)^{2}+0.06(a / b)^{4}\right\} \sqrt{\sec \left(\frac{\pi a}{2 b}\right)}
$$

\section{(Figure 7 is embedded here)}

Fig. 7 Three well-known classic fracture problems, (a) homogeneous finite width center cracked configuration with far field tension [37], (b) homogeneous finite width single edge notch configuration with far field tension[37], (c) bimaterial interface isolated center cracked problem with remotely applied stresses [39].

The values of $F(a / b)$ in Eq. (38) are for a finite width centered crack where $h \rightarrow \infty$. In Fig. 7a when $h / b \geq 3$, the plate is practically regarded as an infinite strip as far as the effects of $h / b$ on the stress intensity factor is concerned [37]. Moreover, the values of $\sigma$ and $a$ need to be updated as the crack propagates during an analysis of a progressive simulation like the lattice model.

\section{(Figure 8 is embedded here)}

Fig. 8 Lattice meshes for the three classic problems to determine stress intensity factors, (a) center cracked lattice mesh to determine the mode 1 stress intensity factor, (b) single edge notch lattice mesh to determine the mode 1 stress intensity factor, (c) heterogeneous bi-material interface center cracked lattice mesh under uniaxial remote tension to determine the modulus of complex stress intensity factor at crack tip.

This problem was simulated in the lattice model trying to obtain the stress intensity factor of the crack tip by using the energy method explained and Eqs. (16) to (23). Fig. 8a shows the lattice mesh employed to simulate the problem. Different simulations were conducted where the height of the configuration, $2 h$, is increased at each simulation to account for the far field tension. If 
the difference between $K_{1}$ obtained by Eq. (37) and that of the lattice model is considered, the error percentage may be regarded as

$$
\operatorname{Error} \%=\frac{\left|K_{1}^{l}-K_{1}^{a}\right|}{K_{1}^{a}} \times 100,
$$

where $K_{1}^{a}$ is the stress intensity factor of the analytical relation, and $K_{1}^{l}$ is that of the lattice. Fig. 9a shows the error percentage of the four lattice simulations with different $a / b$ ratios as the size of the specimen represented by $h / b$ ratio increases. As illustrated, the error plummets as the ratio $h / b$ goes up. The error is just under $40 \%$ for $h / b=1$ while it descends under $1 \%$ when $h / b=4$. As mentioned above, the values obtained by Eq. (38) are for a finite width centered crack where $h \rightarrow \infty$. Also, the plate in Figs. 7a and 8a is practically regarded as an infinite strip for $h / b \geq 3$ according to [37]. Therefore, a large error of $40 \%$ was obtained by the lattice when $h / b=1$, as expected. As it can be seen in Fig. 9a, the error for $h / b=4$ (practically infinite $h$ ) is about $1 \%$. Moreover, it is seen that the error trend is constant as the crack propagates. Thus, it can be confirmed that the implemented lattice model is accurately capable of predicting the mode 1 stress intensity factor for the center cracked problem with far field tension as $h$ increases.

\section{(Figure 9 is embedded here)}

Fig. 9 Error \% variation of Stress Intensity factor between the analytical solution and the lattice model, (a) the centered crack problem under tension, (b) the single edge notch problem under tension, (c) the bi-material interface center cracked problem under direct tension.

\subsubsection{The Single Edge Notch Lattice Simulation}

A single edge notch problem was also considered to evaluate the lattice model's results. Fig. 7b shows the homogeneous finite width single edge notch test specimen with constant thickness on which a far field tensile stress is applied. Mode 1 crack tip stress intensity factor of this problem is again expressed as [37]

$$
K_{1}=\sigma \sqrt{\pi a} F(a / b)
$$

where $a$ is the edge crack length, $\sigma$ is the tensile far field stress, $b$ is the width of the plate or configuration as illustrated in Fig. 7b, and $F(a / b)$ may be expressed as [37] 


$$
F(a / b)=\sqrt{\frac{2 b}{\pi a} \tan \left(\frac{\pi a}{2 b}\right)} \times \frac{0.752+2.02(a / b)+0.37\left(1-\sin \left(\frac{\pi a}{2 b}\right)\right)^{3}}{\cos \left(\frac{\pi a}{2 b}\right)} .
$$

Again, the values of $F(a / b)$ in Eq. (41) are for a finite width single edge notch crack where $h \rightarrow \infty$. Fig. 8b shows the lattice mesh of the single edge notch configuration under direct tension as in Fig. 7b. This lattice mesh was simulated for five different ratios of $2 h / b$. It is observed that as this ratio increases, the error percentage, defined in Eq. (39), declines, as illustrated in Fig. 9b. As expected, the error for $2 h / b=5$ is the lowest which better simulates a farther tensile field than smaller $2 h / b$ ratios. However, the error percentage increases in all cases as the crack propagates through the configuration.

\subsubsection{The Bi-material Interface Center Cracked Lattice Simulation}

To motivate the application of the numerical lattice in characterizing the interface fracture properties of bi-material interfaces such as brick-mortar interface, it may be useful to simulate the bi-material interface center cracked problem. The problem of an isolated finite crack of length $L=2 a$ along the interface between two dissimilar elastic half-spaces subject to two remote stresses $\sigma_{y y}^{\infty}$ and $\sigma_{x y}^{\infty}$ was analytically solved. Fig. 7c illustrates this bi-material interface crack problem.

The complex stress intensity factor at the right hand tip of the crack is expressed as [13], [39]

$$
K=K_{1}+i K_{2}=\left(\sigma_{y y}^{\infty}+i \sigma_{x y}^{\infty}\right)(1+2 i \varepsilon)(\pi L / 2)^{1 / 2} L^{-i \varepsilon},
$$

where $\sigma_{y y}^{\infty}$ and $\sigma_{x y}^{\infty}$ are remotely applied stresses shown in Fig. 7c, $\varepsilon$ is the bi-elastic constant defined in Eq. (11), and $L=2 a$ is the crack length. When the configuration is only under uniaxial direct tension, i.e. $\sigma_{x y}^{\infty}=0$, Eq. (42) reduces to

$$
K=K_{1}+i K_{2}=\sigma_{y y}^{\infty}(1+2 i \varepsilon)(\pi L / 2)^{1 / 2} L^{-i \varepsilon} .
$$

Knowing that $\left|L^{i \varepsilon}\right|=1$ and $\left|K L^{i \varepsilon}\right|=|K|$, the modulus of the complex $K$ is obtained by

$$
|K|=\sigma_{y y}^{\infty} \sqrt{\pi a\left(1+4 \varepsilon^{2}\right)}
$$

which reduces to the well-known relation $\sigma_{y y}^{\infty} \sqrt{\pi a}$ in the absence of mismatch for an infinite plate. Fig. 8c exhibits the lattice mesh with a center crack along the interface to simulate the 
modulus of $K$. The lattice has three phases of mortar, interface and brick which are illustrated by different colors of blue, pink, and black, respectively. Let $L_{x}$ and $L_{y}$ be the width and height of this domain.

To simulate an infinite domain with an isolated crack along its interface, $L_{x}$ and $L_{y}$ have to be sufficiently large to account for the remotely applied stresses. The analysis was conducted for four different values of $L_{x}=L_{y}$. It was observed that by increasing these parameters in the lattice simulation of the problem the error percentage of $|K|$ drops down, indicating that the lattice model can fairly predict the energy release rate and stress intensity factors of bi-material interfaces (Fig. 9c). It is clear that by further increasing the values of $L_{x}=L_{y}$, this error much more decreases.

\subsection{Lattice Simulations of Interface Fracture in Masonry}

Three types of lattice simulations were performed to obtain the energy release rate and fracture properties of brick-mortar interface. They include (i) a pre-notched bi-material four-point bending simulation as shown in Fig. 1, (ii) an unconfined triplet test, Fig. 10, to evaluate the interfacial behavior in mode 2 , and (iii) a direct tension test for mode 1 behavior of the interface.

(Figure 10 is embedded here)

Fig. 10 Unconfined conventional triplet test to evaluate the brick-mortar interface behavior in mode 2.

\subsubsection{The Four Point Bending Simulation}

Because of the symmetry, only one half of the four-point bending beam was accepted in the lattice simulation. For the interface to experience enough length of debonding and delamination, a beam length of $16(\mathrm{in})$ [406.4 ( $\mathrm{mm})]$ with $l=1(\mathrm{in})$ [25.4 ( $\mathrm{mm})]$ and loading points' distance of 15 ( in) [381 ( $\mathrm{mm})$ ] was considered for the simulations (see Fig. 1). A mortar layer of 1.5 ( in) [38.1 ( $\mathrm{mm})]$ was overlaid on a brick layer of $1(\mathrm{in})[25.4(\mathrm{~mm})]$ thick. Since the crack propagates into the brick layer at early stages of the simulation when the thickness of brick is greater than that of mortar, a thicker mortar layer was considered in this study for the fourpoint bending simulation as it results in an interface crack propagation during the analysis which was the main purpose of the simulation to get the interface fracture properties. An analysis was 
conducted to select the type of mesh and a regular square mesh, which is actually a random mesh with no degree of randomness, gave more consistent results than regular triangular and random meshes. This is probably due to the fact that in a regular square mesh, the crack flanks behind the tip in the continuum mesh are straight surfaces while in a regular triangular mesh with the hexagonal configuration of the continuum mesh (Fig. 3a), the crack surfaces have a zigzag pattern affecting the results of interfacial energy release rate and other fracture quantities. Fig. 11 illustrates the lattice mesh and the boundary conditions of the notched symmetric composite beam under the four-point bending used in the lattice analysis. Since the top layer above the crack includes a notch, it is essentially stress free experiencing rigid body motion behind the crack tip. Moreover, because of both opening and sliding of the upper mortar layer relative to the lower brick layer, there is a mixed mode failure in the interface.

\section{(Figure 11 is embedded here)}

Fig. 11 The lattice mesh and the boundary conditions of a notched symmetric composite beam used in the lattice analysis under the four-point bending. This figure belongs to an increment with a propagated crack during the analysis with a magnification factor of 300 .

Fig. 12 shows the fracture results of the lattice configuration shown in Fig. 11. Since the moment is constant between the loading points, the values of energy release rate, loading phase angle, and stress intensity factors remain fairly constant and steady state during crack propagation. It should be noted that the phase angle is insensitive to the distance from the crack tip, $r$, which makes the fracture quantities of mode 1 and mode 2, e.g. the stress intensity factor and the fracture energy, independent of distance $r$. In Fig. 12, some points on the graph of $G$ and consequently $K_{1}$ and $K_{2}$ have lower values especially for $50 \leq a \leq 120(\mathrm{~mm})$, resulting in stepwise oscillations. These points are related to those interface elements which fail 'immediately' after the failure of the previous neighboring interface strut, resulting in a lower load value in the load-displacement diagram. As shown in Fig. 12b, the distribution of the phase angle, $\hat{\psi}$, varies between $45^{\circ}$ and $55^{\circ}$, meaning that the fracture is a mixed mode, as $\hat{\psi}=0^{\circ}$ and $\widehat{\psi}=90^{\circ}$ indicate pure mode 1 and pure mode 2 , respectively.

(Figure 12 is embedded here) 
Fig. 12 The four-point bending simulation results from lattice analysis, (a) the energy release rate, $G$, with respect to crack length, (b) the phase angle, $\hat{\psi}$, at different distances from the tip with respect to crack length $\left(b_{t}\right.$ is related to square size in the mesh), (c) the stress intensity factor for mode 1 , (d) the stress intensity factor for mode 2.

Moreover, Fig. 12a compares the energy release rate values of the lattice simulation with those of an analytical solution for a notched four-point bending beam obtained by Charalambides et al. [18]. This analytical approach is based on Euler-Bernoulli beam theory and plane strain conditions. The energy release rate may be expressed in terms of the applied moment $M$, material properties, and cross-section geometries of the two layers as

$$
G=\frac{M^{2}\left(1-v_{2}^{2}\right)}{2 E_{2}}\left(\frac{1}{I_{2}}-\frac{\lambda}{I_{c}}\right)
$$

where $M=P l / 2 b$, with $P$ being the total load, $\lambda=E_{2}\left(1-v_{1}{ }^{2}\right) / E_{1}\left(1-v_{2}{ }^{2}\right), I_{c}=h_{1}{ }^{3} / 12+$ $\lambda h_{2}{ }^{3} / 12+\lambda h_{1} h_{2}\left(h_{1}+h_{2}\right)^{2} / 4\left(h_{1}+\lambda h_{2}\right)$, and $I_{2}=h_{2}{ }^{3} / 12$. In Fig. 12a, the error percentage of $G$ between the lattice and analytical results, similar to the one defined in Eq. (39), starts from $17 \%$ for crack length of 1.09 (in) $[28(\mathrm{~mm})]$ and reduces to $7 \%$ when crack length is around 4.24 (in) $[108(\mathrm{~mm})]$. It then increases when the crack tip approaches the roller support. As can be seen in Fig. 12a, the energy release rate values predicted by the lattice model are lower than those approximated by the analytical solution. This is possibly due to the fact that the interface struts of the lattice, as discussed in Section 3, have no rotational stiffness similar to truss elements where there is no bending stiffness contribution, while in the Euler-Bernoulli beam theory which was employed in determining the analytical solution of Eq. (45), bending stiffness plays an important role, resulting in stiffer solution with higher $G$ values.

The change of fracture quantities with respect to mesh refinement should also be considered. Fig. 13a,b illustrates the change of the energy release rate and the phase angle for a fine mesh which is about five times finer than the coarse mesh. As shown in Fig. 13a, G is relatively insensitive to the mesh size, while $\hat{\psi}$ experiences a small reduction from a coarser mesh to a finer one for a pre-notched four-point bending beam.

(Figure 13 is embedded here) 
Fig. 13 The lattice simulations of the four-point bending beam test (a) mesh sensitivity of the energy release rate, $G$, (b) mesh sensitivity of the phase angle, $\widehat{\psi}$, (c) the energy release rate for pure tension in mode 1 , (d) the energy release rate for pure shear in mode 2.

As discussed earlier, the value of Dundur's parameter $\beta$ for concrete and masonry is sufficiently small such that a zero $\beta$ hypothesis can be assumed. For $\beta=0$, the components of the energy release rate, G, can be related to the stress intensity factor for mode 1 and mode 2 as [22]

$$
G_{1}=\frac{K_{1}^{2}}{E_{*}}, G_{2}=\frac{K_{2}^{2}}{E_{*}},
$$

where $\mathrm{G}=G_{1}+G_{2} . G_{1}$ and $G_{2}$ can be regarded as the work performed by normal and shear tractions on the interface moving through the crack surface displacements of mode 1 opening and mode 2 slip, respectively [22]. When $\beta \neq 0$, this decomposition does no longer exist. Fig. $\mathbf{1 3 c}, \mathbf{d}$ shows the variations of energy release rate for mode 1 and mode 2 for the lattice analysis of Fig. 11. As can be seen in the figure, as the crack length increases, mode 2 slightly increases with crack extension, which is also evident in Fig. 12b for the phase angle.

The values of $G, G_{1}$, and $G_{2}$, shown in the figures, are the critical energy release rates when a crack extension happens in the lattice model (Fig. 11). These critical values are equivalent to the area under the traction-separation curve of a cohesive zone interface model. The pink interface struts in Fig. 11 fail one by one in an unzipping manner. If it is assumed that the cohesive zone, between the fully-damaged and undamaged part of the interface, always contains one interface strut, then each point in Fig. 12a, which is due to the energy released by removing that single interface strut in the cohesive zone, is equivalent to the area beneath the traction-separation law after initiation of damage. Constant steady-state variation of $G$ in Fig. 12a is a useful characteristic of the simulation in Fig. 11 which can give a consistent result, as an estimate, for the required fracture energy parameter for the traction-separation cohesive zone model. Determining the parameters of a bilinear traction-separation law, which is the simplest cohesive zone model, is nontrivial and conducting an experimental program to measure them is very difficult, but necessary. These values of energy release rate obtained by the lattice may provide an 'estimate' of the fracture energy parameter for the traction-separation cohesive zone models. However, it should be emphasized that the 'true' traction-separation behavior is only obtained through experimental tests. 
(Figure 14 is embedded here)

Fig. 14 The energy release rate of the cohesive zone determined by the lattice analysis in the traction-separation plane.

Fig. 14 illustrates the energy release rate of the cohesive zone determined by the lattice analysis for a mixed mode. The shaded triangular area in the bilinear traction-separation law is equivalent to the $G$ value obtained by the lattice model. The area of the left triangle, which belongs to the linear behavior of the cohesive zone, can directly be determined by the linear properties and the maximum traction value.

\subsubsection{The Unconfined Triplet Simulation}

Furthermore, the unconfined triplet test, which is a double lap shear test in masonry studies, was considered for the lattice simulations. Fig. 15 shows the triplet boundary conditions with the propagated crack length of delamination from the lattice analysis.

(Figure 15 is embedded here)

Fig. 15 The lattice mesh and the boundary conditions of a triplet configuration used in the lattice analysis of the unconfined triplet simulation. This figure belongs to an increment with a propagated crack during the analysis.

Fig. 16 shows the fracture results of the lattice configuration shown in Fig. 15. Fig. 16a illustrates the variation of $G$ with respect to the crack length for one interface, which exhibits a constant steady-state trend. Again, each point on this graph is related to the failure of one strut at the cohesive zone. In Fig. 16b, the variation of $\widehat{\psi}$ at different distances from the crack tip, $r$, is shown, which is insensitive to this distance. This feature is promising in decomposing the energy release rate and the modulus of stress intensity factor into mode 1 and mode 2. Fig. 16c and Fig. 16d compare the stress intensity factors for mode 1 and 2, respectively. As shown, the unconfined triplet simulation indicates that this is not a pure shear process as there are some values on Fig. 16c for mode 1 separation. However, the shear failure is dominant as compared to the tension one.

(Figure 16 is embedded here) 
Fig. 16 The unconfined triplet simulation results from lattice analysis, (a) the energy release rate, $G$, with respect to crack length, (b) the phase angle, $\hat{\psi}$, at different distances from the tip with respect to crack length $\left(b_{t}\right.$ is related to square size in the mesh), (c) the stress intensity factor for mode 1 , (d) the stress intensity factor for mode 2.

Fig. 17a,b shows the mesh sensitivity of the lattice unconfined triplet simulation comparing the energy release rate and the phase angle for a mesh which is about four times finer than the coarse mesh. The values of energy release rate are fairly insensitive to the mesh refinement, while the loading phase angle is slightly different for the coarser and finer mesh. However, this small difference may be neglected.

\section{(Figure 17 is embedded here)}

Fig. 17 The lattice simulations of an unconfined triplet test, (a) mesh sensitivity of the energy release rate, $G$, (b) mesh sensitivity of the phase angle, $\hat{\psi}$, (c) the energy release rate for pure tension in mode 1 , (d) the energy release rate for pure shear in mode 2.

Fig. 17c,d also illustrates the critical energy release rates for mode 1 and 2 of the unconfined triplet test shown in Fig. 15. As expected, the values of $G$ for mode 1 is negligible compared to those of mode 2. $G_{2}$ obtained by the lattice simulation of the triplet can be utilized in the traction-separation law, as shown in Fig. 14, for mode 2 of the interface failure. In other words, the continuum traction-separation cohesive zone model can be informed by the fracture energy results obtained from the discrete lattice model.

\subsubsection{The Direct Tension Simulation}

Another lattice simulation conducted was the tension test of the brick-mortar interface. The boundary condition and configuration of the tensile simulation is shown in Fig. 18 with a propagated crack at one interface. The brick and mortar configuration is similar to that of the triplet test, which can be used here for the tension since the crack only happens at one interface.

\section{(Figure 18 is embedded here)}

Fig. 18 The lattice mesh and the boundary conditions of a masonry configuration used in the lattice analysis of the direct tensile simulation. This figure belongs to an increment with a propagated crack during the analysis. 
The fracture properties of this simulation are illustrated in Fig. 19. This analysis also provides steady state values for the energy release rates of the interface as the crack propagates. Comparing Fig. 19c and Fig. 19d indicates that the major contributing mode in the interface failure is mode 1 , as expected. The phase angle is again fairly insensitive to the distance from the crack tip as exhibited in Fig. 19b.

\section{(Figure 19 is embedded here)}

Fig. 19 The direct tension simulation results from lattice analysis, (a) the energy release rate, $G$, with respect to crack length, (b) the phase angle, $\hat{\psi}$, at different distances from the tip with respect to crack length $\left(b_{t}\right.$ is related to square size in the mesh), (c) the stress intensity factor for mode 1, (d) the stress intensity factor for mode 2.

The mesh sensitivity of the lattice simulation was also examined for the case of direct tension. The energy release rate and the loading phase angle obtained from the finer mesh have the same variation and trend when compared with those of the coarser mesh, as shown in Fig. 20a,b. Fig. 20c,d also exhibits the dominance of mode 1 critical energy release rate over that of mode 2 which is an intrinsic characteristic of the direct tension test.

(Figure 20 is embedded here)

Fig. 20 The lattice simulations of the direct tension test, (a) mesh sensitivity of the energy release rate, $G$, (b) the phase angle, $\hat{\psi},(\mathrm{c})$ the energy release rate for pure tension in mode $1,(\mathrm{~d})$ the energy release rate for pure shear in mode 2.

At a prescribed phase angle, $\widehat{\psi}$, the maximum loading amplitude or the critical energy release rate, $G$, that an interface can sustain without decohesion is the toughness of that interface at $\widehat{\psi}$, which is noted as $\Gamma(\widehat{\psi})[40]$

$$
G=\Gamma(\widehat{\psi})
$$

The interface toughness is not a single material parameter, but rather it is a function of the mode mixity at the interface depending on the mode of loading. It is possible to extract the interface toughness curve from these three categories of lattice simulations, i.e. the pre-notched four-point bending beam, the unconfined triplet simulation, and the uniaxial tension test. Fig. 21 illustrates the toughness function for the masonry interface obtained by the lattice model. It indicates that 
the interface toughness is approximately a linear function for the interface behavior in direct tension, in four-point bending, and in double lap shear conditions.

(Figure 21 is embedded here)

Fig. 21 Fracture toughness curve, $\Gamma(\hat{\psi})$, for a brick-mortar interface obtained from the three lattice simulations

\section{Concluding Remarks}

A 2-D plane strain lattice model was implemented to obtain relevant fracture properties of masonry interface at different loading and boundary conditions. The Voronoi diagram was used to discretize a continuum domain into polygons, or particles. Plane frame elements with three degrees of freedom at each node were chosen because of their capability to better capture the crack pattern in continuum domains than truss elements, and also due to their added computational stability. The strength properties of brick, mortar, and interface were then projected on top of the lattice struts based on their coordinates on the domain to simulate a threephase boundary value problem. The simulation of fracture was performed with a 'linear elastic' analysis of the lattice under loading and removing one element at a time from the mesh which exceeds a certain failure criterion in tension, compression, and shear. Failure of brick and mortar were expressed in terms of a tension cut-off and compression-cap. Shear failure criteria were neglected for brick and mortar elements since the main focus of this study was to evaluate the brick-mortar interface fracture properties, and with the simulations conducted, their shear failure envelopes were not activated. The failure criterion for the brick-mortar interface was also determined based on a combination of experimental measurements and numerical parametric calculations. This failure function has a compressive cap which lets diagonal interface struts in a regular triangular lattice fail for triplet tests under high normal confinements. The concepts of interfacial fracture mechanics were used along with the implementation of the lattice model to determine the fracture properties of the brick-mortar interface.

The concept of total potential energy of the lattice model was used to determine the energy release rate of an interface strut failure while the crack develops. In the method, the energy released during the crack growth is mainly determined by considering the change in the global stiffness matrix of the mesh with respect to crack length change. Comparison of the analytical results with the numerical solutions of the three benchmark problems and also the pre-notched 
bi-material four-point bending simulation validates the capability of the lattice in predicting the energy release rate and stress intensity factor of crack problems in homogeneous and heterogeneous solids. In the case of classic benchmark problems, the lattice gives better results once the dimensions of the mesh are large enough to assure that the stresses are remotely applied. It should be mentioned that the lattice square mesh was considered for fracture analyses of the crack problems rather than using random or regular triangular meshes. Unlike the random and regular triangular meshes which provide intrinsic angled or zigzag crack surfaces producing irregular oscillations in the values of energy release rate and stress intensity factor, the square mesh can generate straight crack surfaces along the interface which is itself an almost direct straight surface between brick and mortar. The irregular oscillations observed in the values of $G$ and $|K|$ with the regular triangular mesh were discarded by using the square mesh.

Three types of masonry lattice simulations were performed to obtain the energy release rate and fracture properties of brick-mortar interface. Each of these simulations exhibit different failure mode mixity. In the pre-notched bi-material four-point bending simulation, the interface experiences a mixed mode failure with almost the same contribution of mode 1 and mode 2 , as depicted in Fig. 12b by the phase angle variations. As expected, the dominant interfacial failure mode in the unconfined triplet test is mode 2, while mode 1 has the major contribution in interface failure for the direct tension simulation (See Fig. 16b and Fig. 19b). The values of $G$, $G_{1}$, and $G_{2}$ rendered by the lattice simulations are the critical energy release rates when a crack extension happens. These critical values are equivalent to the area under the traction-separation curve of a cohesive zone interface model after the damage initiates. Determining the parameters of a bilinear traction-separation law is nontrivial and it is very difficult to conduct an experimental program to measure them. The implemented micro-level lattice model is a promising approach to determine energy release quantities that can readily be used in a mesolevel continuum traction-separation interface model. Knowing that the values of $G$ are the critical ones, it is possible to determine the interface fracture toughness curve based on these three types of simulations. The present approach can also be used to obtain the fracture energy for a variety of interfaces of other bi-materials, relating the discretized microstructure to the continuum meso-structure. 


\section{ACKNOWLEDGEMENTS}

The authors would like to acknowledge the support of this research effort by the US National Science Foundation under the NSF project No. 1100971 named "Interface Mechanics of Masonry Panels under Bi-axial Loading". The authors would also like to appreciate the valuable comments of the anonymous reviewer. Opinions expressed in this paper are those of the authors and do not necessarily reflect those of the sponsor.

\section{REFERENCES}

[1] R. Goodman, R. Taylor, L. Brekke, A Model for the Mechanics of Jointed Rock, in: J. Soil Mech. Found. Div. Proc. Am. Soc. Civ. Eng., 1968: pp. 637-659.

[2] A.W. Page, Finite Element Model for Masonry, J. Struct. Div. 104 (1978) 1267-1285.

[3] H.R. Lotfi, P.B. Shing, Interface Model Applied to Fracture of Masonry Structures, J. Struct. Eng. 120 (1994) 63-80.

[4] P.B. Lourenço, Computational Strategies for Masonry Structures, Delft University Press, 1996.

[5] P.B. Lourenço, J.G. Rots, Multisurface Interface Model for Analysis of Masonry Structures, J. Eng. Mech. 123 (1997) 660-668.

[6] I. Carol, C.M. López, O. Roa, Micromechanical Analysis of Quasi-brittle Materials Using Fracture-based Interface Elements, Int. J. Numer. Methods Eng. 52 (2001) 193-215. doi:10.1002/nme.277.

[7] K.J. Willam, I. Rhee, B. Shing, Interface Damage Model for Thermomechanical Degradation of Heterogeneous Materials, Comput. Methods Appl. Mech. Eng. 193 (2004) 3327-3350. doi:10.1016/j.cma.2003.09.020.

[8] G. Alfano, E. Sacco, Combining Interface Damage and Friction in a Cohesive-zone Model, Int. J. Numer. Methods Eng. 68 (2006) 542-582. doi:10.1002/nme.1728.

[9] A. Caballero, K.J. Willam, I. Carol, Consistent Tangent Formulation for 3D Interface Modeling of Cracking/Fracture in Quasi-brittle Materials, Comput. Methods Appl. Mech. Eng. 197 (2008) 2804-2822. doi:10.1016/j.cma.2008.01.011.

[10] E. Sacco, J. Toti, Interface Elements for the Analysis of Masonry Structures, Int. J. Comput. Methods Eng. Sci. Mech. 11 (2010) 354-373. doi:10.1080/15502287.2010.516793.

[11] N.I. Muskhelishvili, Some Basic Problems on the Mathematical Theory of Elasticity, Translated by J.R.M. Radok, Noordhoof, Groningen, The Netherlands, 1953.

[12] M.L. Williams, The Stresses around a Fault or Crack in Dissimilar Media, Bull. Seismol. Soc. Am. 49 (1959) 199-204.

http://www.bssaonline.org/content/49/2/199.abstractln/home/bellecave/THESIS/Biblio/BIBLIOGRAPHY/ Williams 1959 - The stresses around a fault or crack in dissimilar media.pdf.

[13] J.R. Rice, G.C. Sih, Plane Problems of Cracks in Dissimilar Media, J. Appl. Mech. 32 (1965) 418-423. doi:10.1115/1.3625816.

[14] a. H. England, A Crack Between Dissimilar Media, J. Appl. Mech. 32 (1965) 400-402. doi:10.1115/1.3625813.

[15] F. Erdogan, Stress Distribution in Bonded Dissimilar Materials With Cracks, J. Appl. Mech. 32 (1965) 403- 
410. doi:10.1115/1.3625814.

[16] J.R. Rice, Elastic Fracture Mechanics Concepts for Interfacial Cracks, J. Appl. Mech. (1988).

[17] D.M. Parks, A Stiffness Derivative Finite Element Technique for Determination of Crack Tip Stress Intensity Factors, Int. J. Fract. 10 (1974) 487-502.

[18] P.G. Charalambides, J. Lund, A.G. Evans, R.M. McMeeking, A Test Specimen for Determining the Fracture Resistance of Bimaterial Interfaces, J. Appl. Mech. 56 (1989) 77-82. doi:10.1115/1.3176069.

[19] P.P.L. Matos, R.M. McMeeking, P.G. Charalambides, M.D. Drory, A Method for Calculating Stress Intensities in Bimaterial Fracture, Int. J. Fract. 40 (1989) 235-254. doi:10.1007/BF00963659.

[20] D.M. Parks, The virtual crack extension method for nonlinear material behavior, Comput. Methods Appl. Mech. Eng. 12 (1977) 353-364. doi:10.1016/0045-7825(77)90023-8.

[21] a. G. Evans, M. Rühle, B.J. Dalgleish, P.G. Charalambides, The fracture energy of bimaterial interfaces, Metall. Trans. A. 21 (1990) 2419-2429. doi:10.1007/BF02646986.

[22] J.. Hutchinson, Z. Suo, Mixed Mode Cracking in Layered Materials, Adv. Appl. Mech. 29 (1992) 63-191.

[23] J. Dundurs, Elastic interaction of dislocations with inhomogeneities, Math. Theory Dislocations. (1969) 70115. http://129.105.37.34/refbase/files/Dundurs.pdf.

[24] O. Büyüköztürk, K.-M. Lee, Assessment of Interfacial Fracture Toughness in Concrete Composites, Cem. Concr. Compos. 15 (1993) 143-151. doi:10.1016/0958-9465(93)90003-R.

[25] Z. Suo, J.W. Hutchinson, Sandwich Test Specimens for Measuring Interface Crack Toughness, Mater. Sci. Eng. A107 (1989) 135-143. doi:10.1016/0921-5093(89)90382-1.

[26] A. Hrennikoff, Solution of Problems of Elasticity by the Framework Method, J. Appl. Mech. 12 (1941) 169175.

[27] J.E. Bolander Jr, S. Saito, Fracture Analyses Using Spring Networks with Random Geometry, Eng. Fract. Mech. 61 (1998) 569-591.

[28] E. Schlangen, E.J. Garboczi, New Method for Simulating Fracture Using an Elastically Uniform Random Geometry Lattice, Int. J. Eng. Sci. 34 (1996) 1131-1144.

[29] J. van Mier, Concrete Fracture, A Multiscale Approach, CRC Press, 2013. doi:10.1201/b12968.

[30] E. Schlangen, J.G.M. van Mier, Experimental and Numerical Analysis of Micromechanisms of Fracture of Cement-based Composites, Cem. Concr. Compos. 14 (1992) 105-118. doi:10.1016/0958-9465(92)90004-F.

[31] A. Mohammadipour, Interface Fracture in Masonry Composites: A Lattice Approach, (Doctoral Dissertation), University of Houston, 2015.

[32] A.H. Mohammadipour, K.J. Willam, A. Ayoub, Experimental Studies of Brick and Mortar Composites Using Digital Image Analysis, in: Proc. 8th Int. Conf. Fract. Mech. Concr. Concr. Struct. Fram., Toledo; Spain, 2013: pp. 183-192.

[33] K.J. Willam, A.H. Mohammadipour, R. Mousavi, A.S. Ayoub, Failure of Unreinforced Masonry under Compression, in: Struct. Congr. 2013 Bridg. Your Passion with Your Prof. - Proc. 2013 Struct. Congr., Pittsburgh, PA; United States, 2013: pp. 2949-2961.

[34] ASTM, Standard Test Method for Splitting Tensile Strength of Masonry Units. ASTM C 1006-84., (2001).

[35] O.C. Zienkiewicz, R. Taylor, J. Zhu, The Finite Element Method: Its Basis and Fundamentals, 6th ed., Elsevier Butterworth-Heinemann, 2005.

[36] T. Anderson, Fracture Mechanics: Fundamentals and Applications, 3rd ed., Taylor \& Francis, 2005.

[37] H. Tada, P.C. Paris, G.R. Irwin, The Stress Analysis of Cracks Handbook, 3rd ed., ASME Press, New York, 2000.

[38] W.T. Koiter, Note on the Stress Intensity Factor for Sheet Strips with Cracks under Tensile Loads, Delft, The Netherlands, 1965.

[39] J.W. Hutchinson, M.E. Mear, J.R. Rice, Crack Paralleling an Interface Between Dissimilar Materials, J. 
Appl. Mech. 54 (1987) 828-832. doi:10.1115/1.3173124.

[40] J.-S. Wang, Z. Suo, Experimental determination of interfacial toughness curves using Brazil-nutsandwiches, Acta Metall. Mater. 38 (1990) 1279-1290. doi:10.1016/0956-7151(90)90200-Z. 
Figure 1

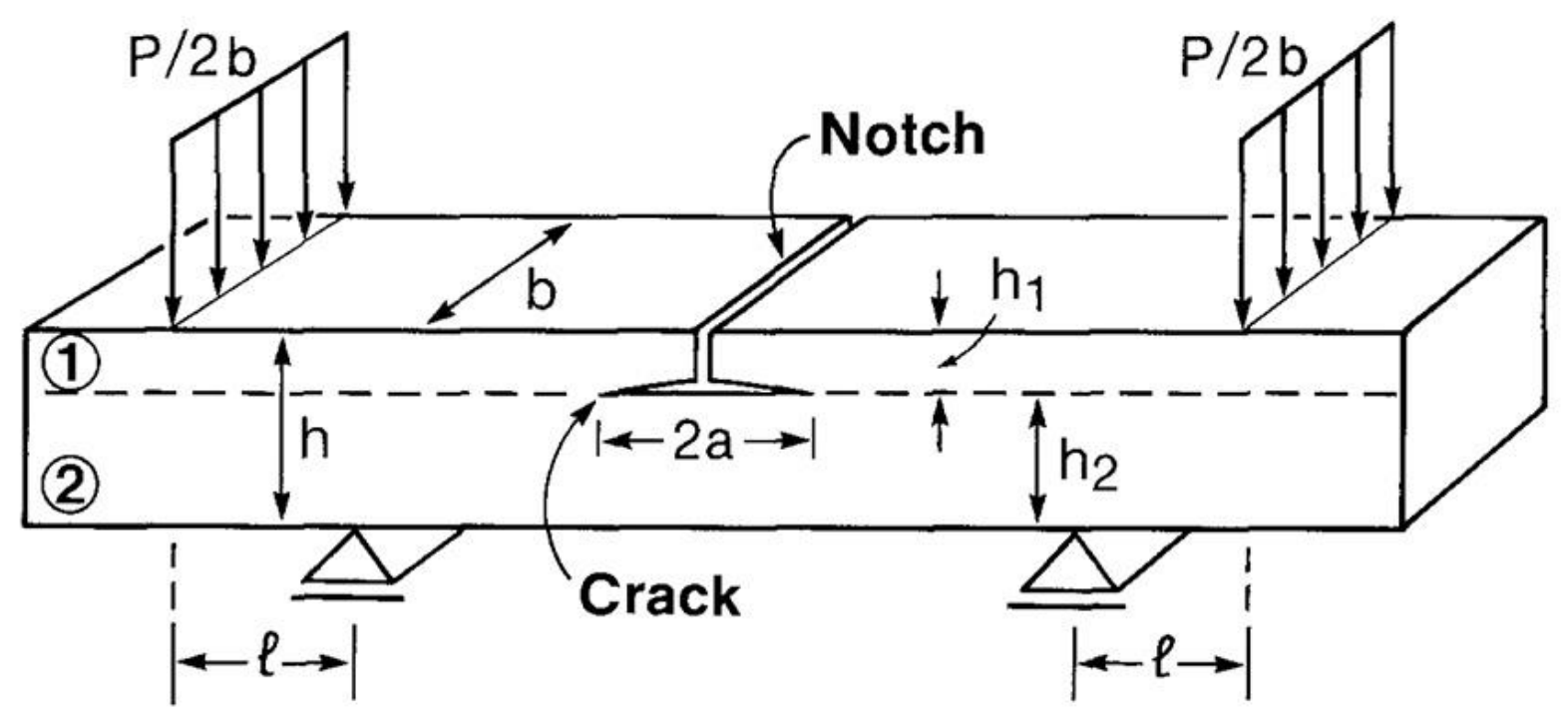


Figure 2

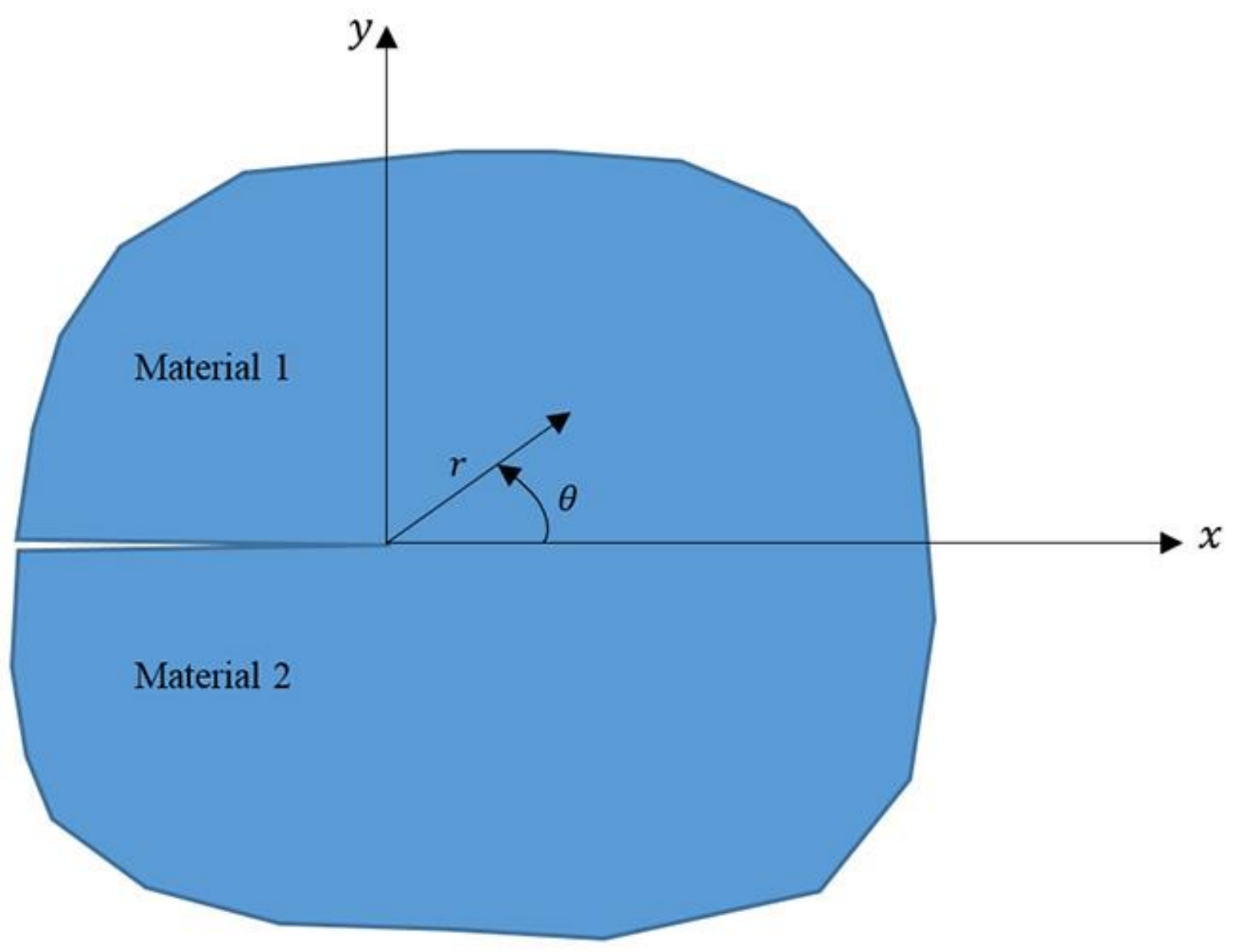


Figure 3

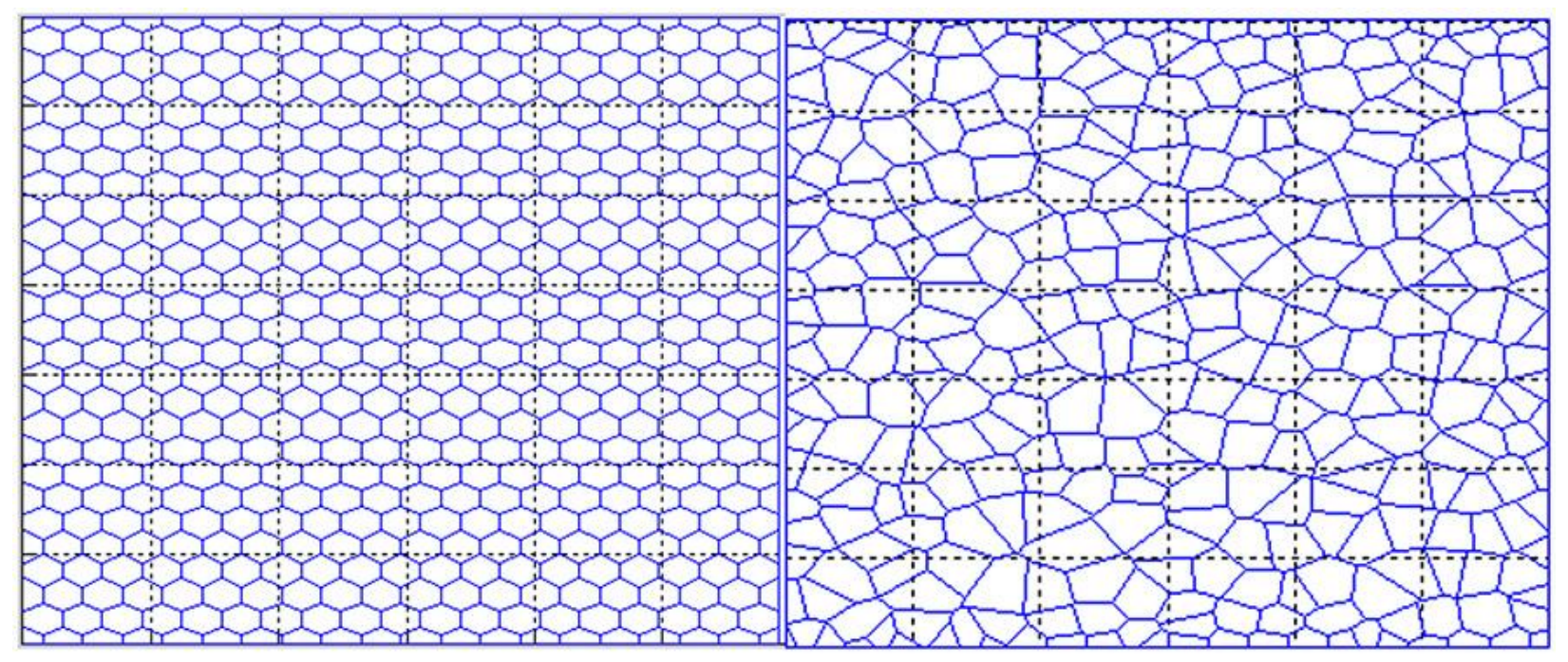


Figure 4

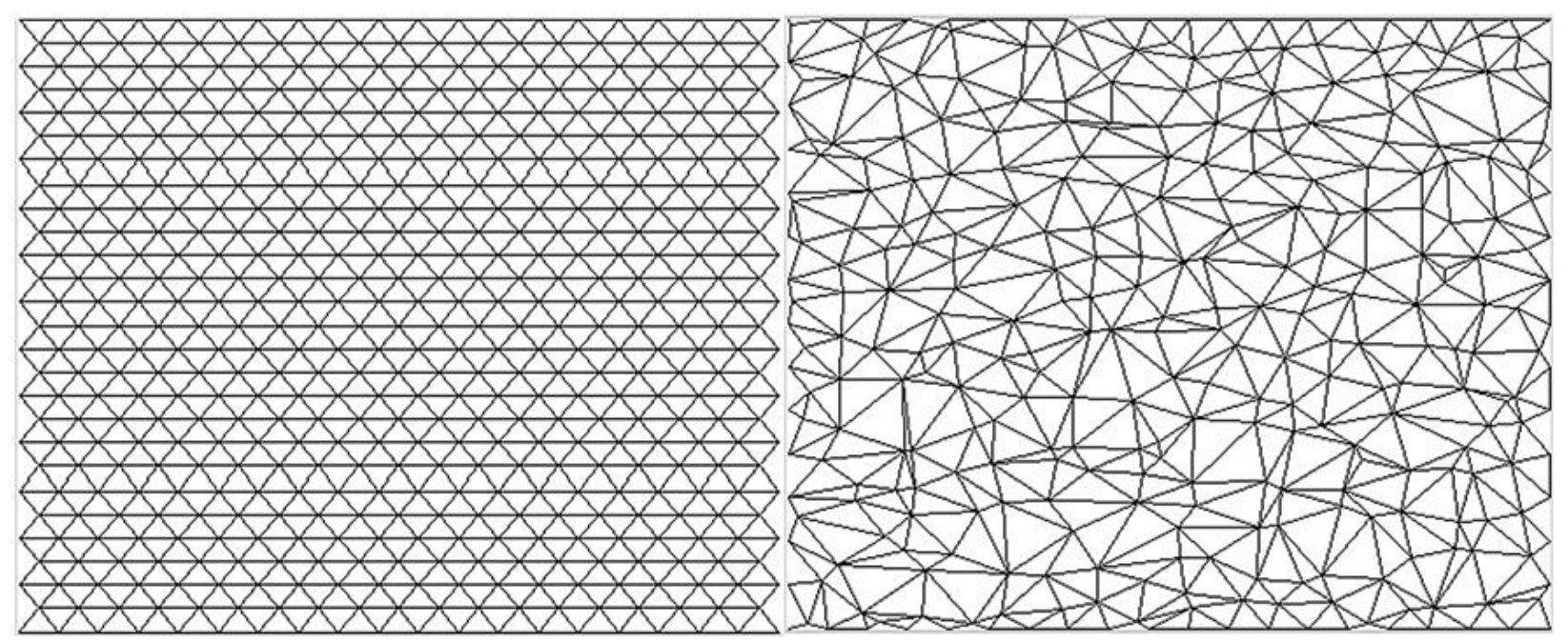


Figure 5

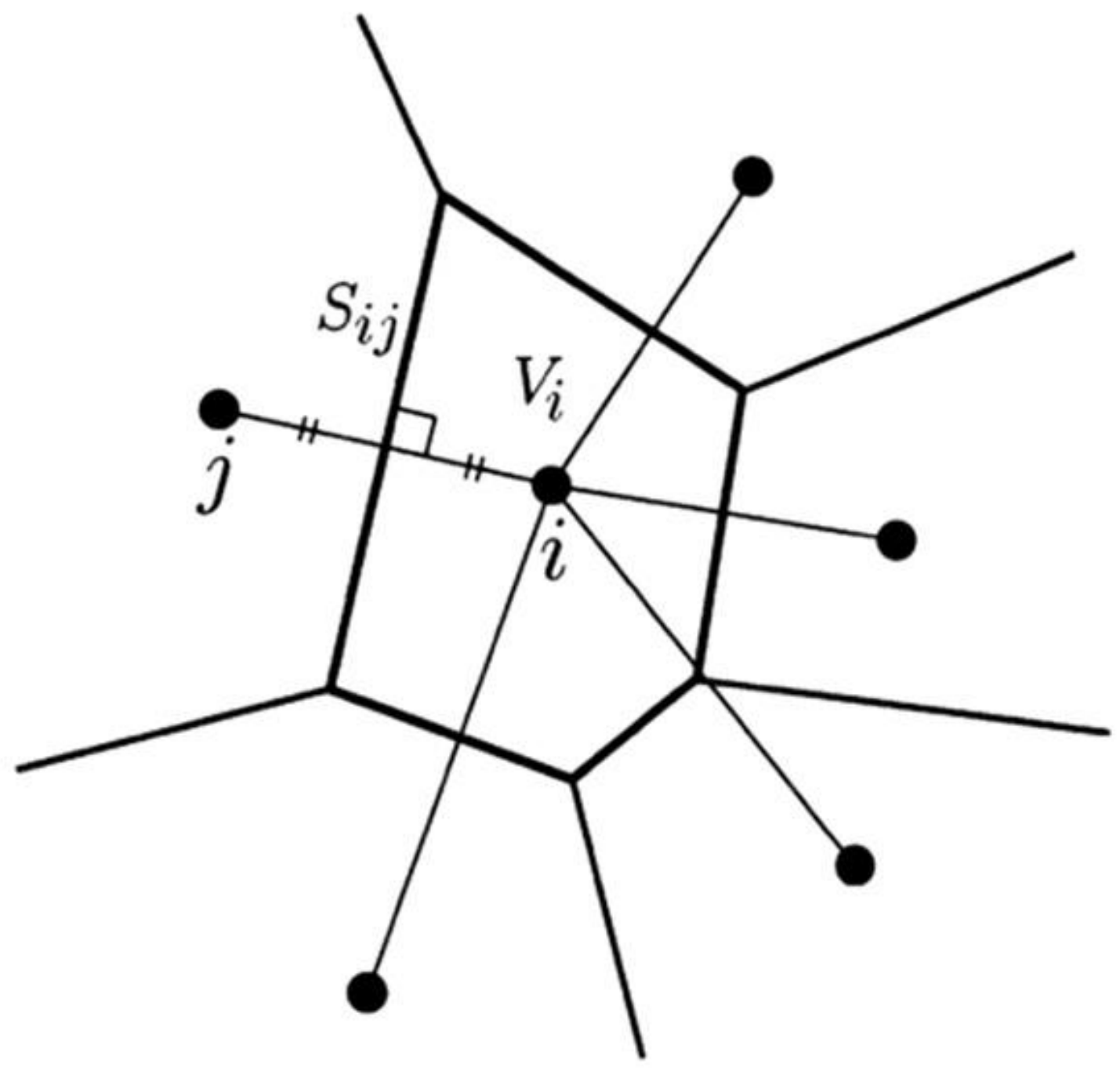


Figure 6

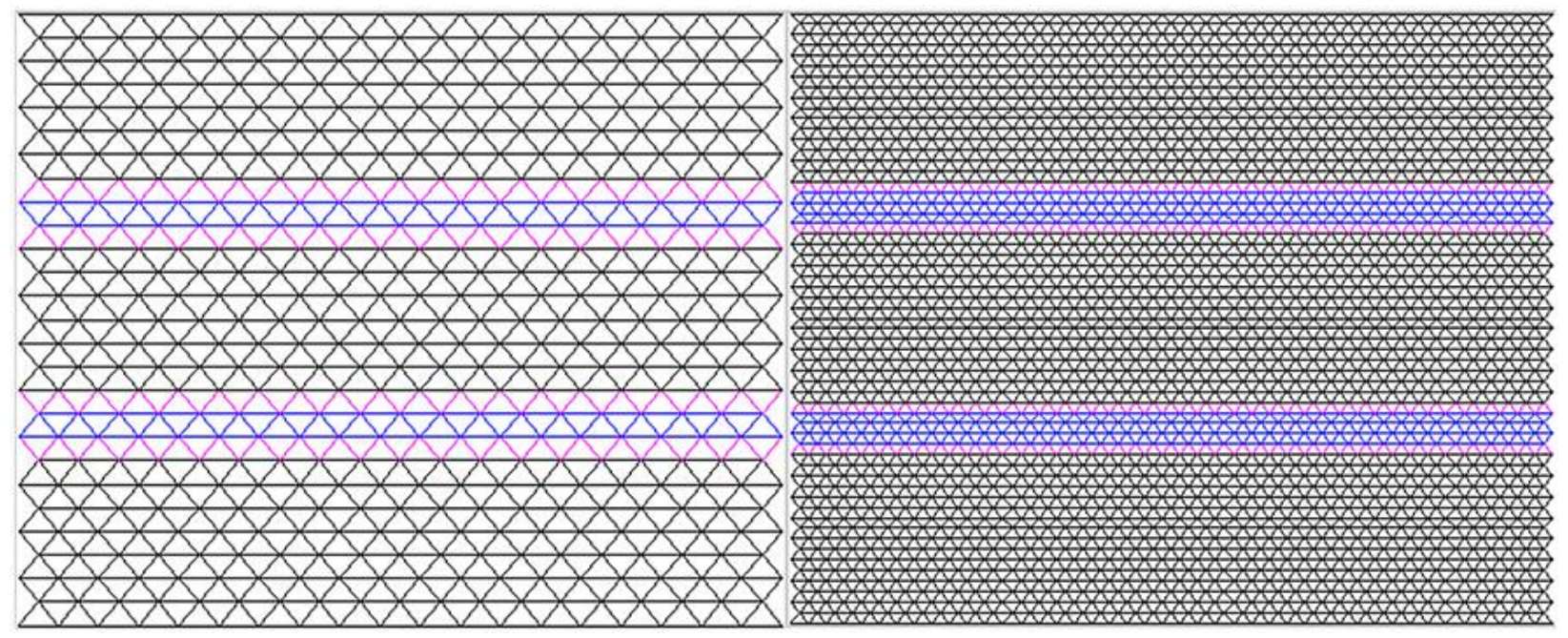


Figure 7

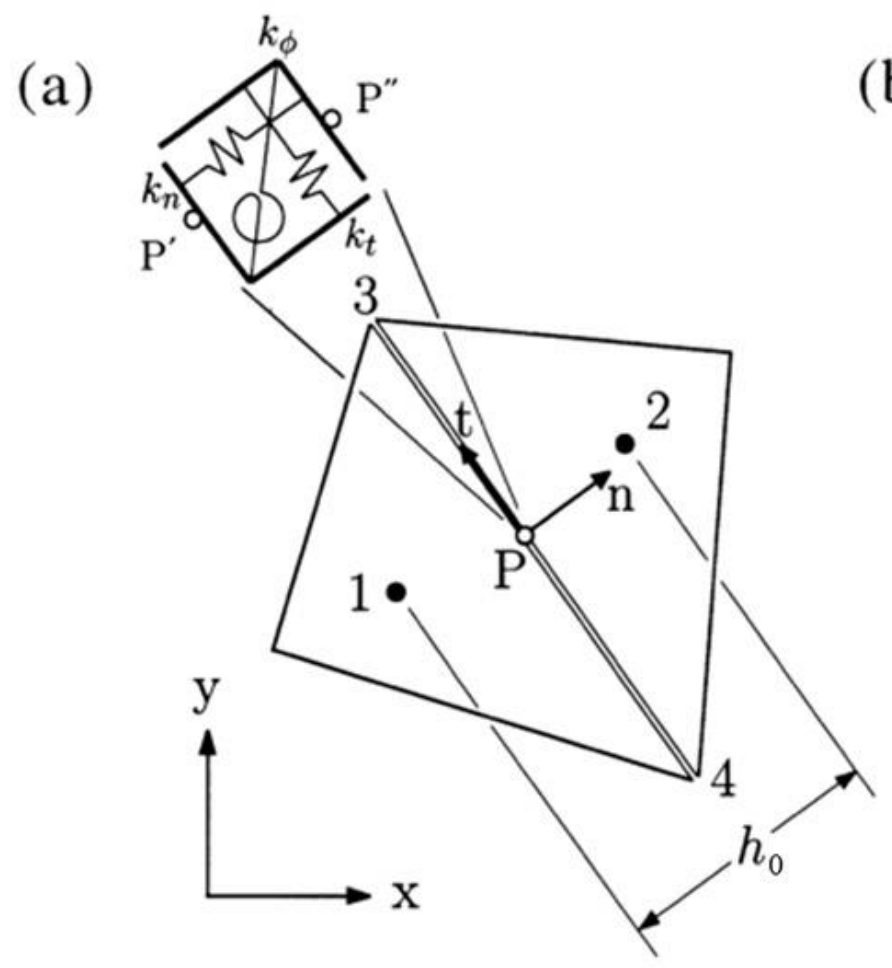

(b)

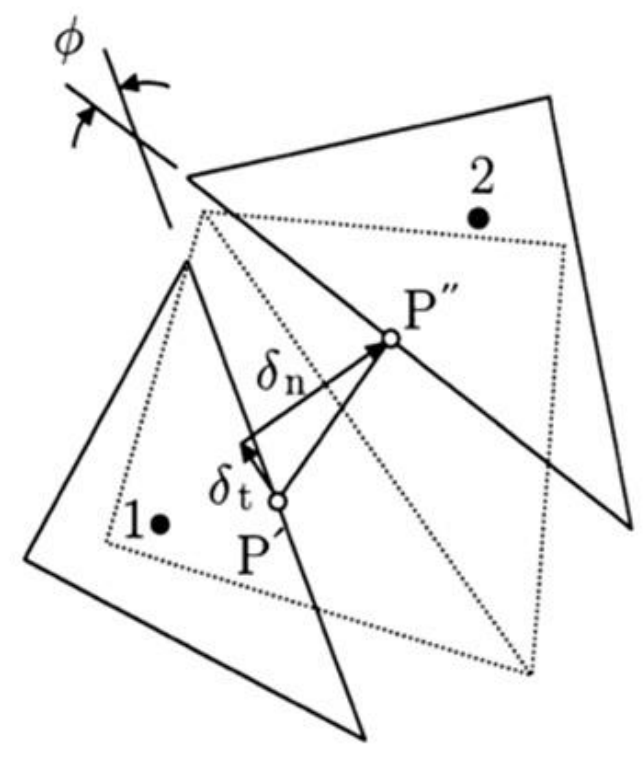


Figure 8

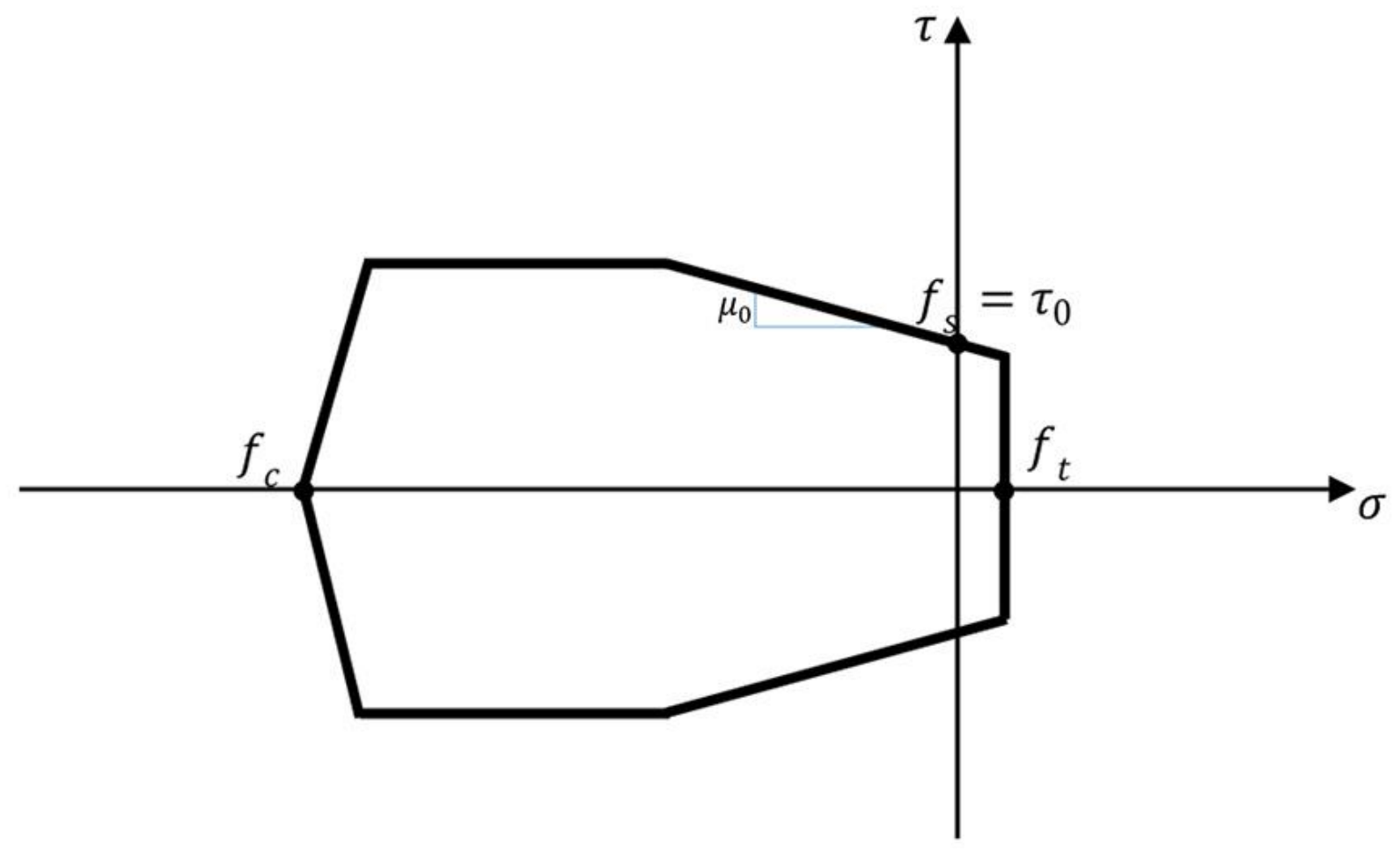


Figure 9

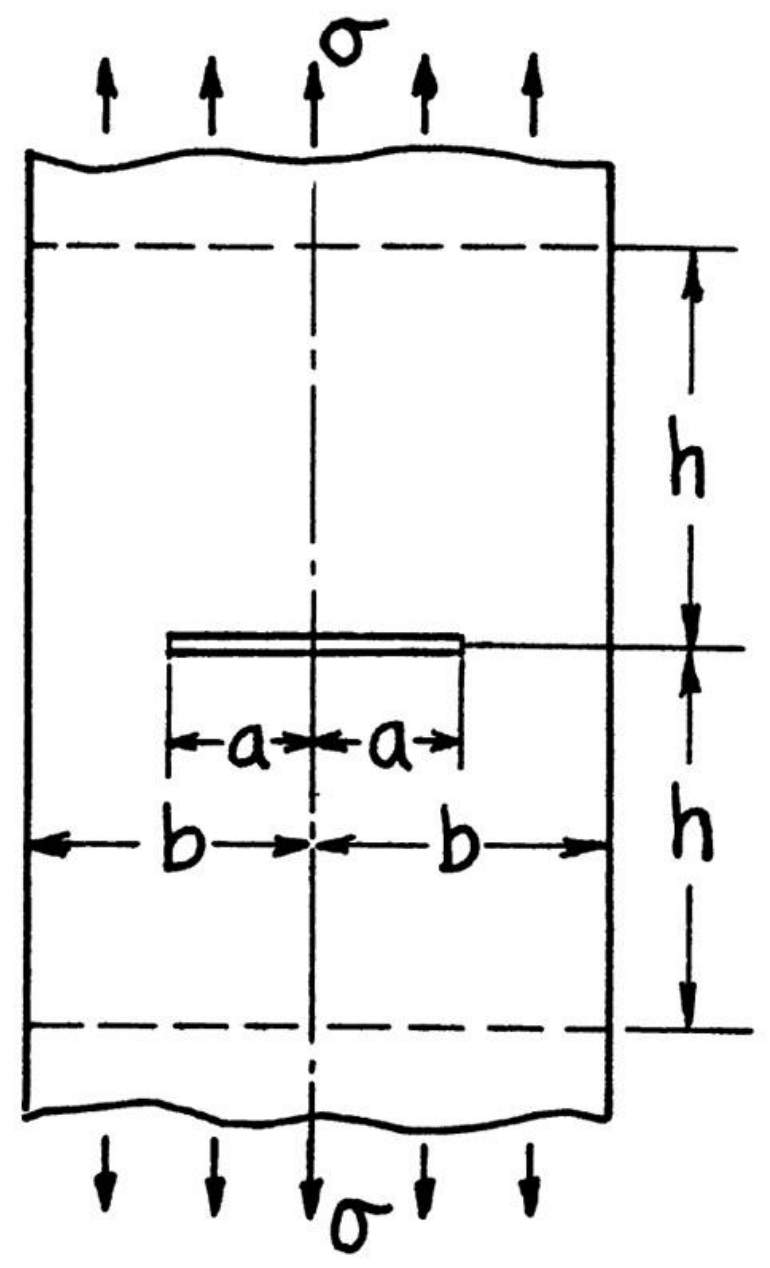


Figure 10

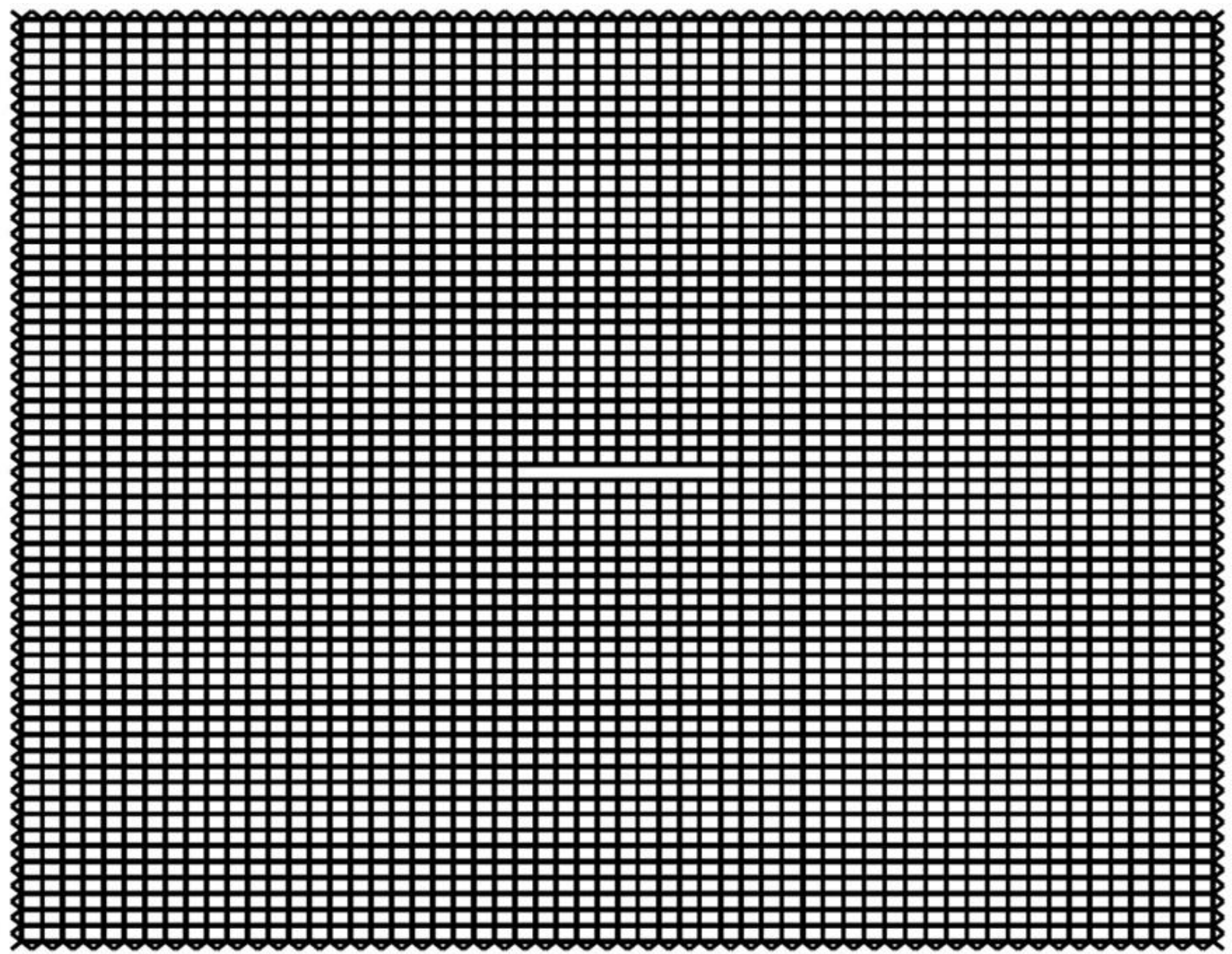


Figure 11

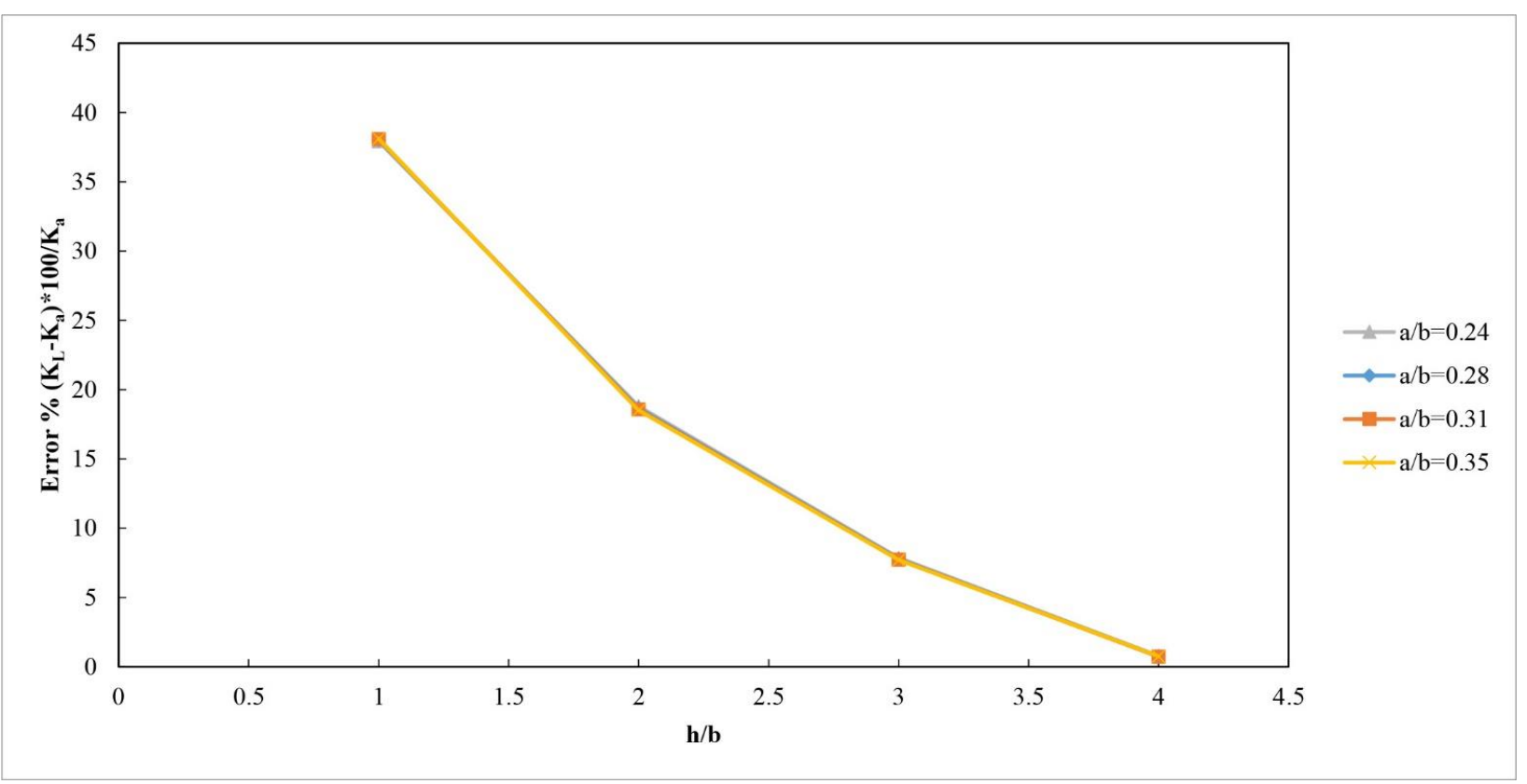


Figure 12

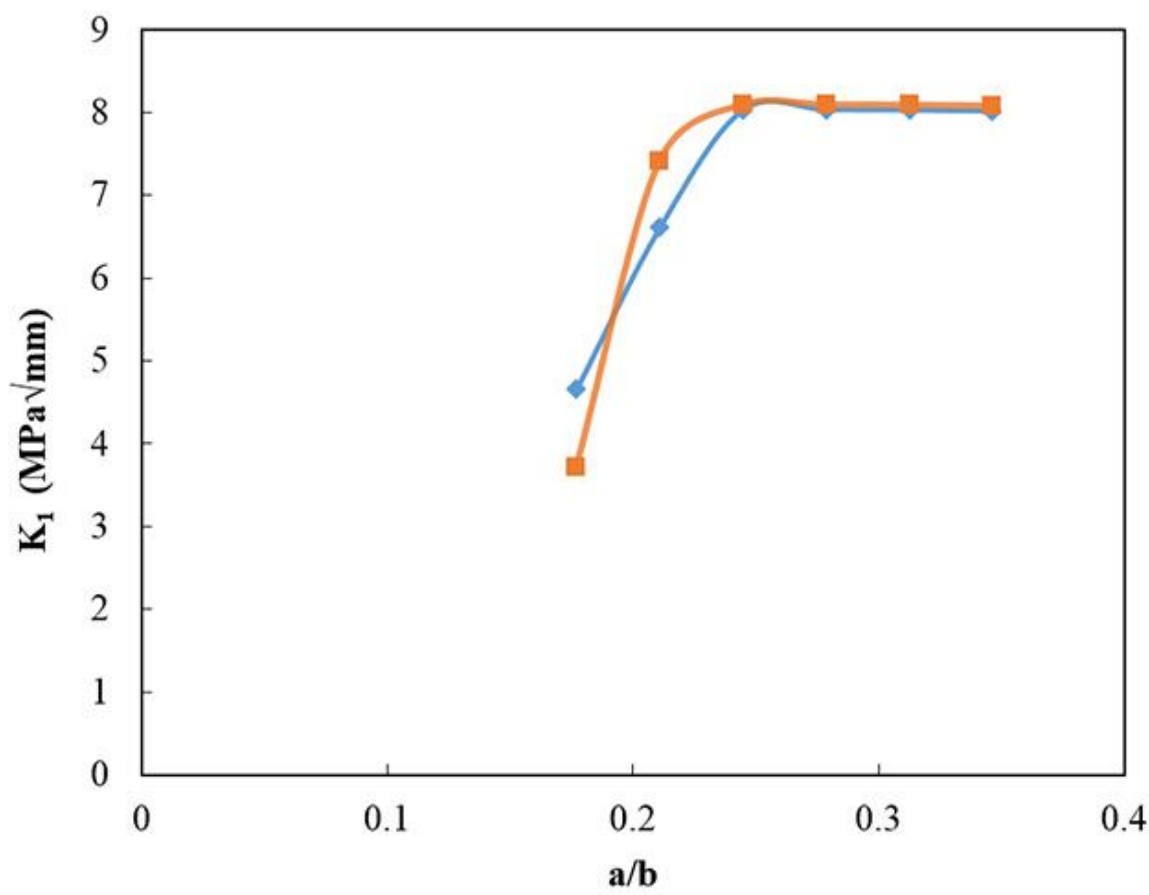

$\multimap$ Lattice Model

- - Analytical Solution 
Figure 13

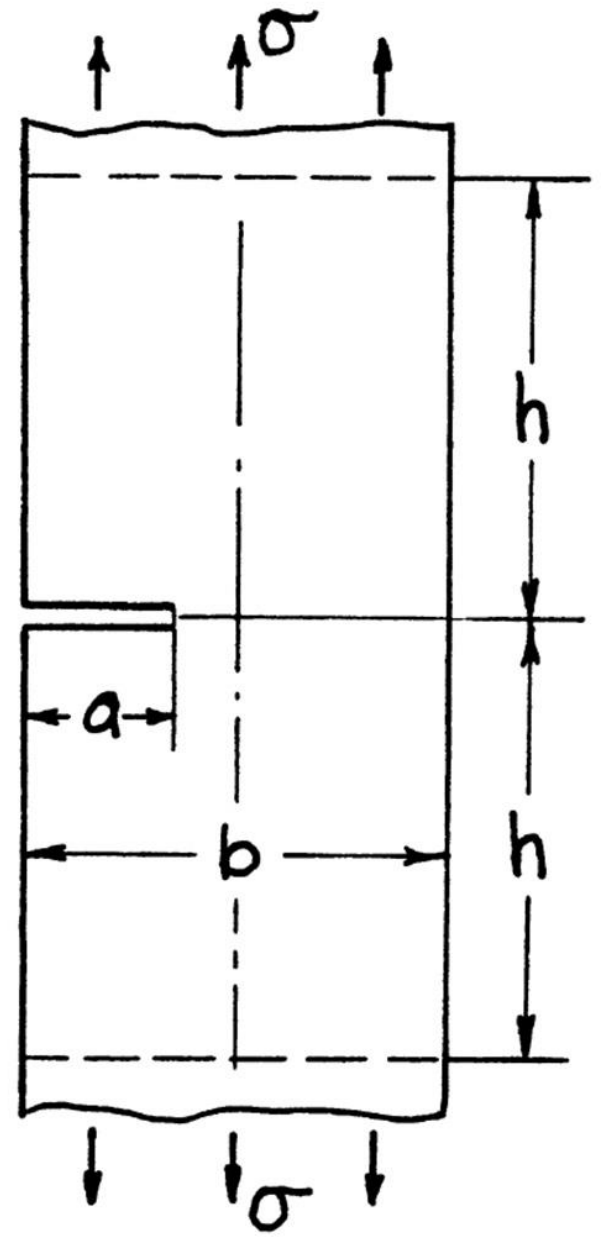

44 
Figure 14

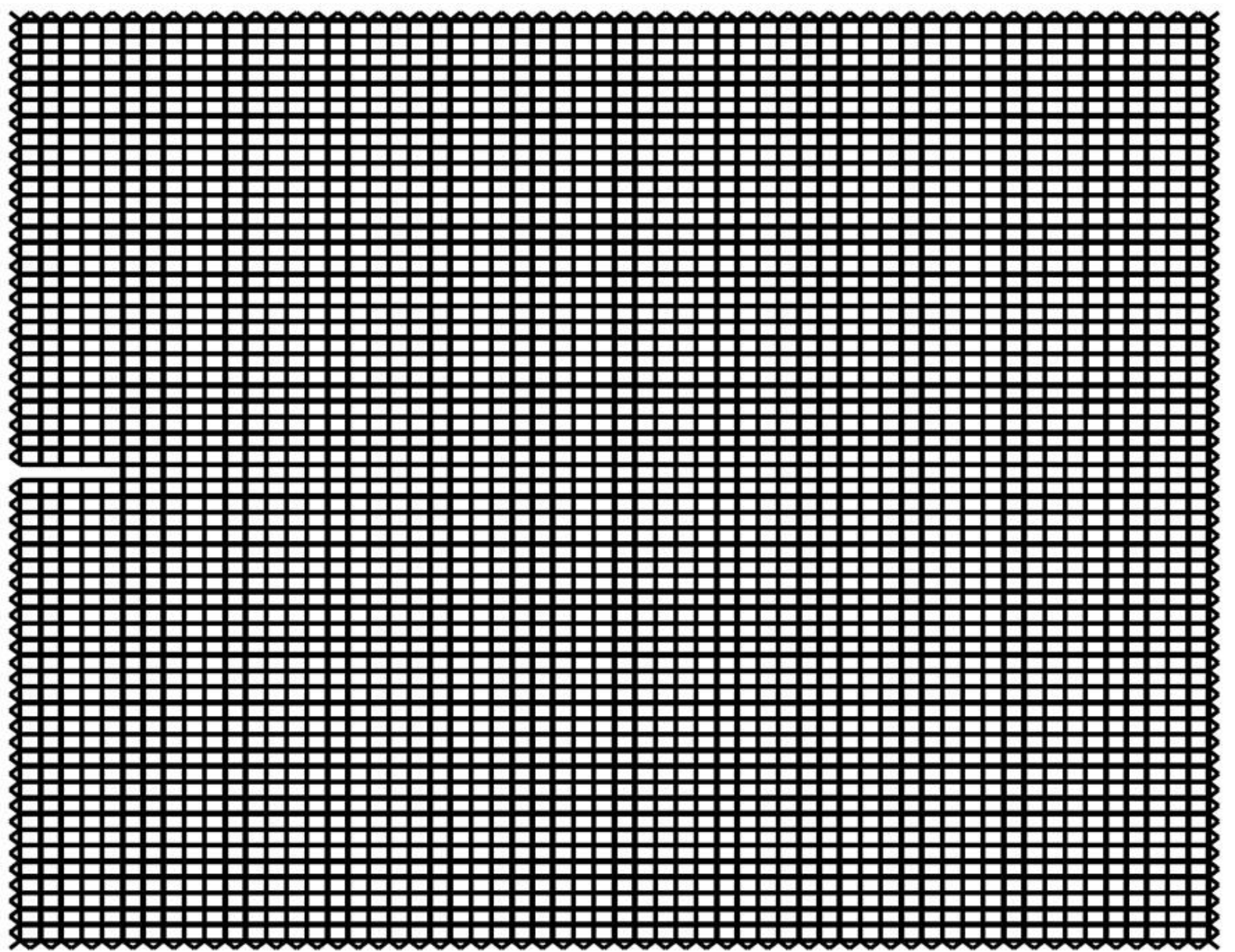


Figure 15

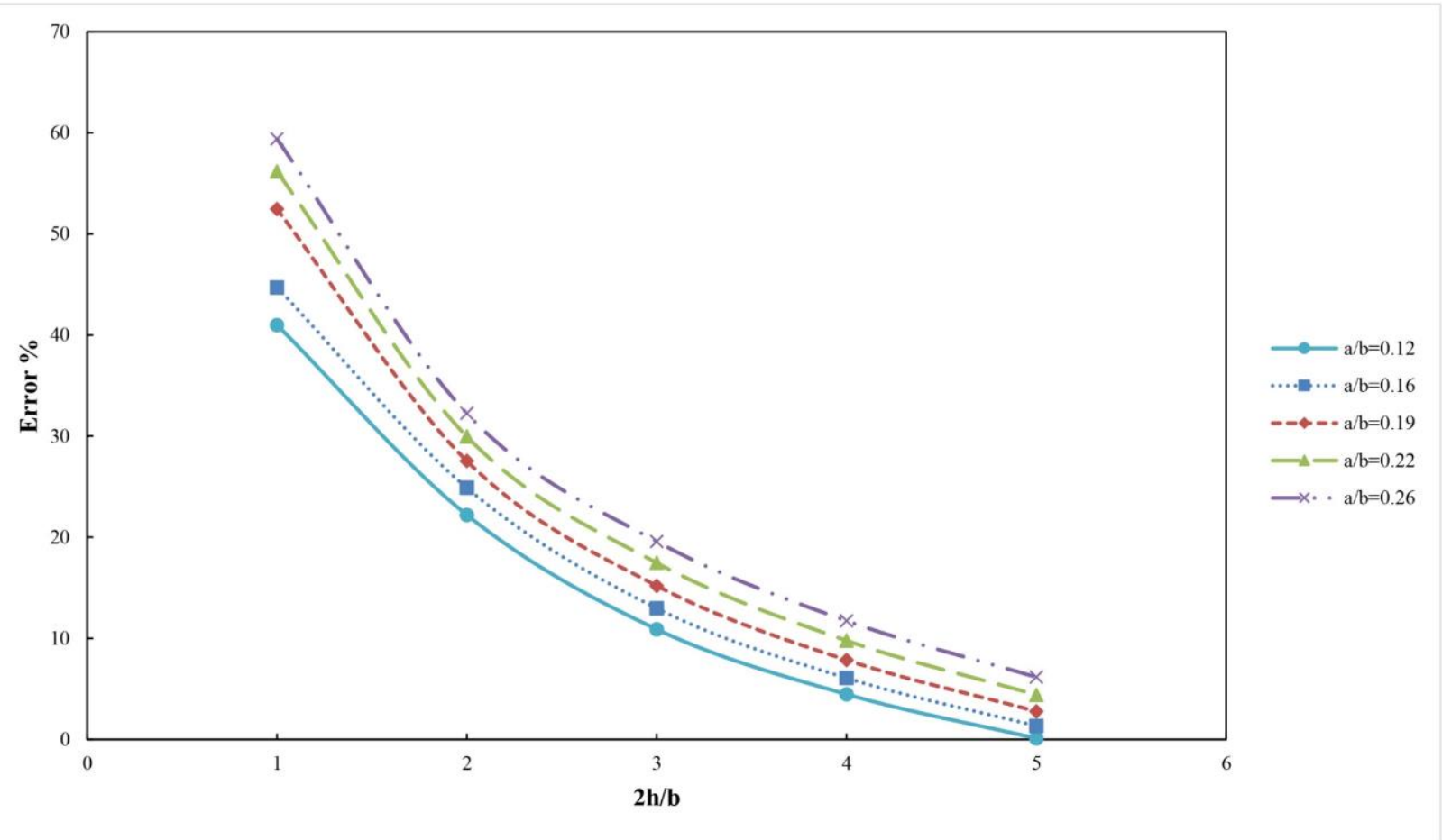


Figure 16

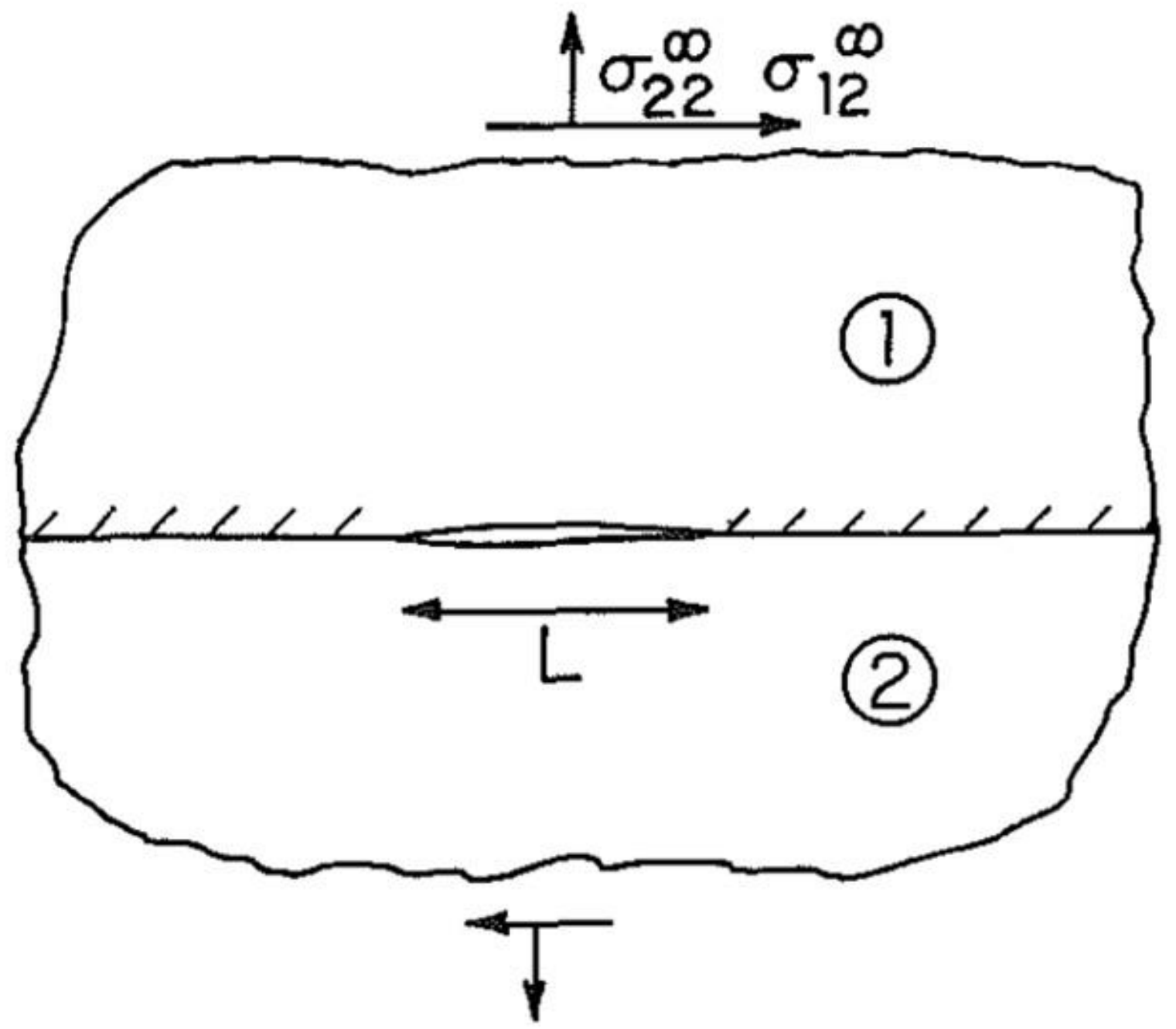


Figure 17

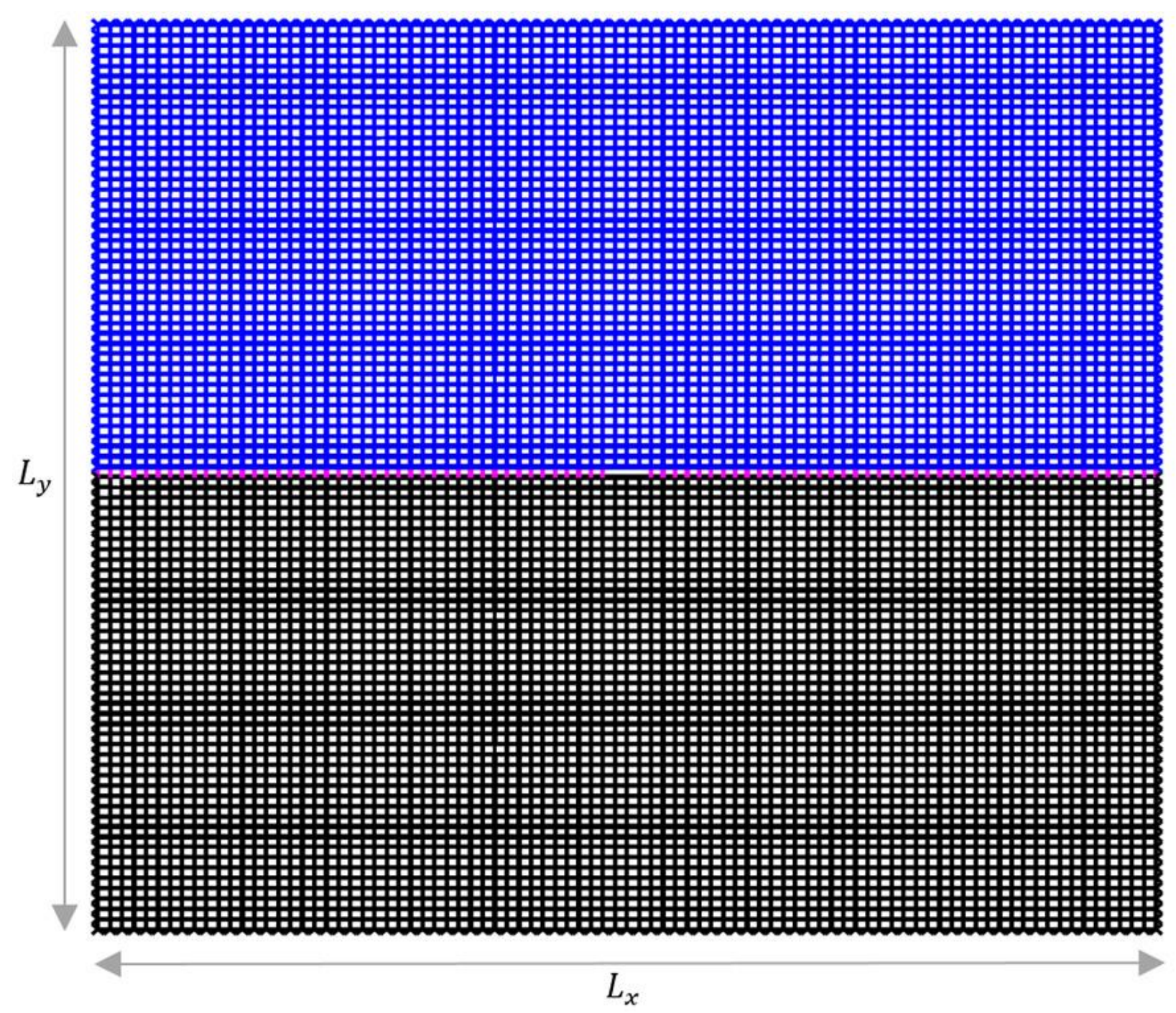


Figure 18

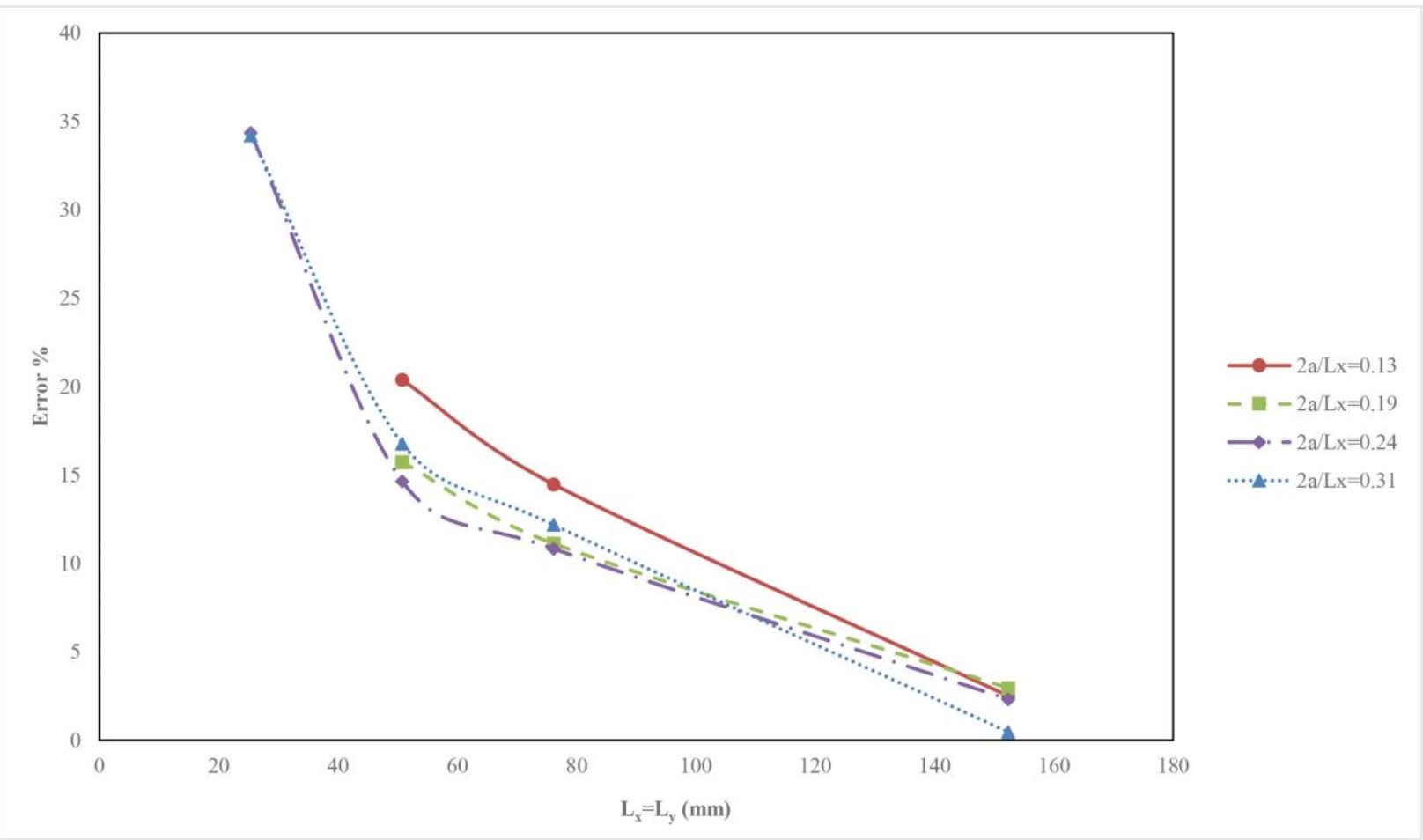


Figure 19

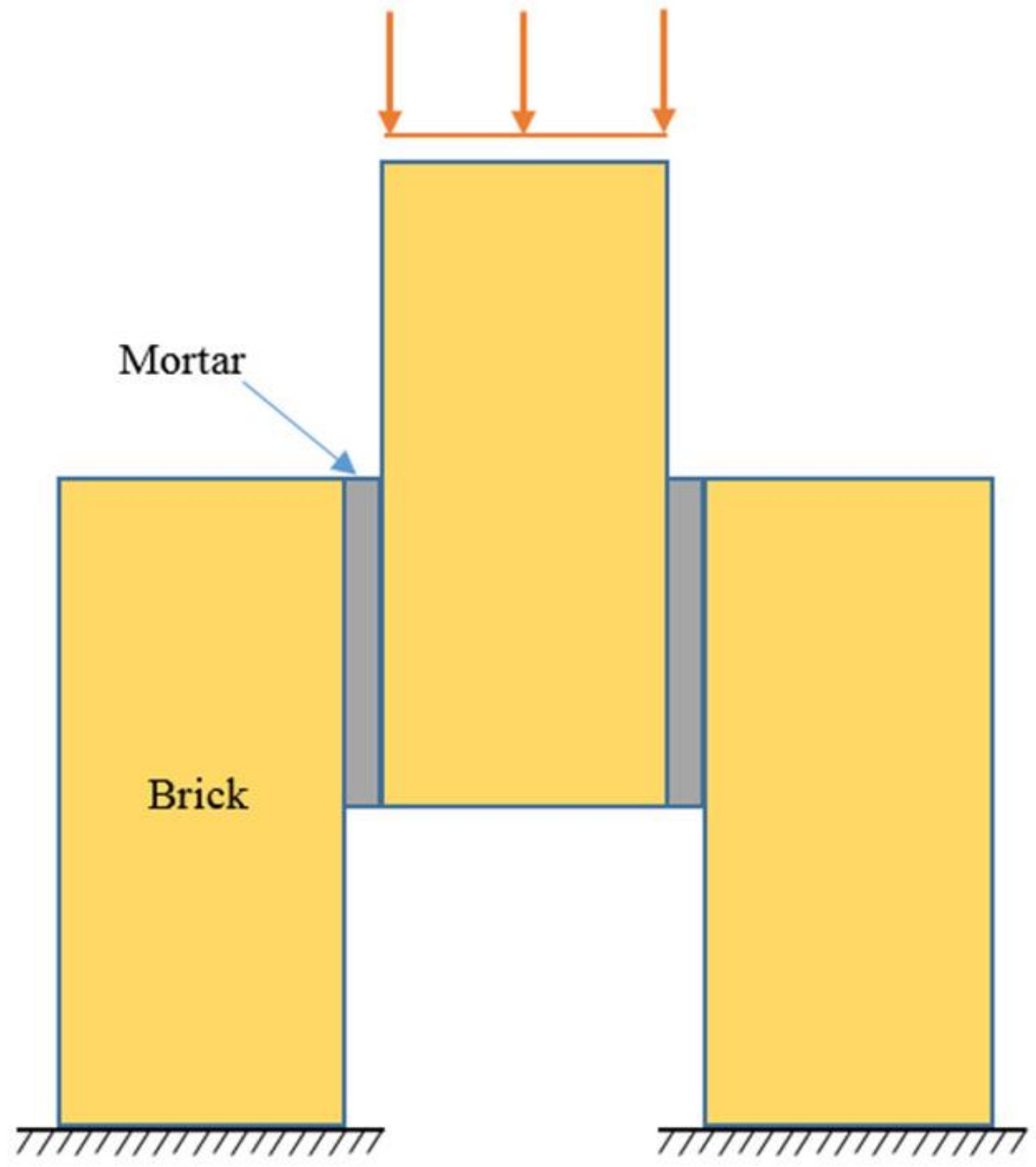


Figure 20

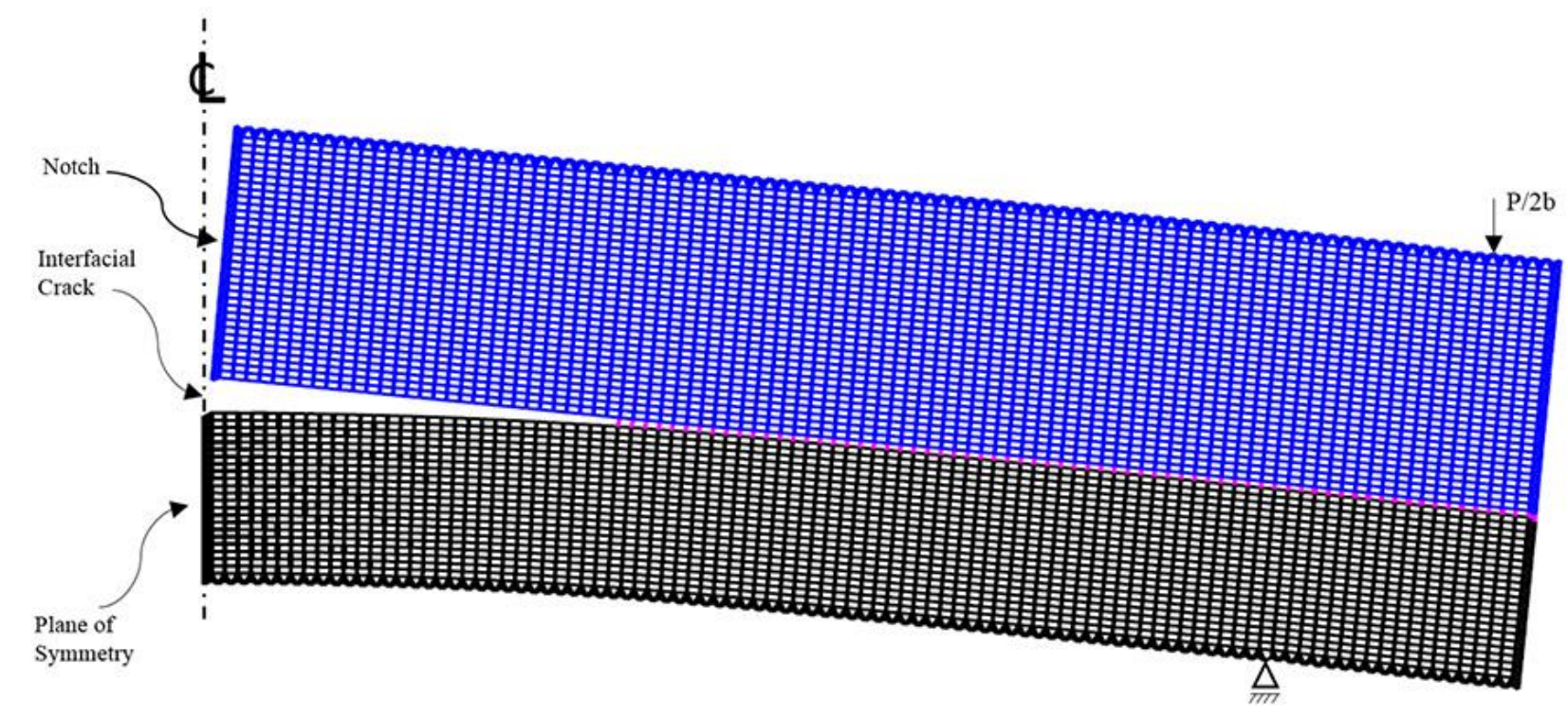


Figure 21
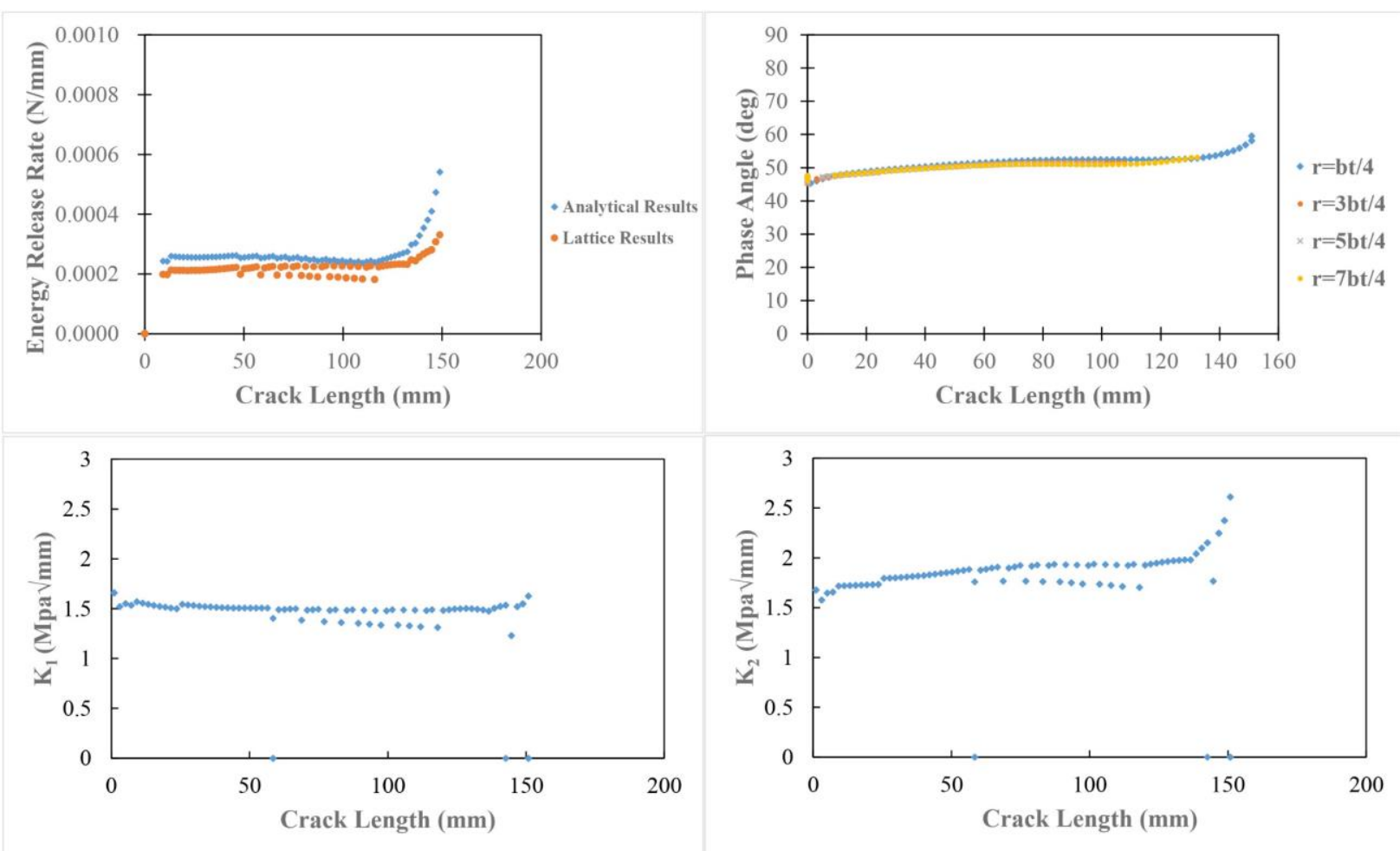
Figure 22
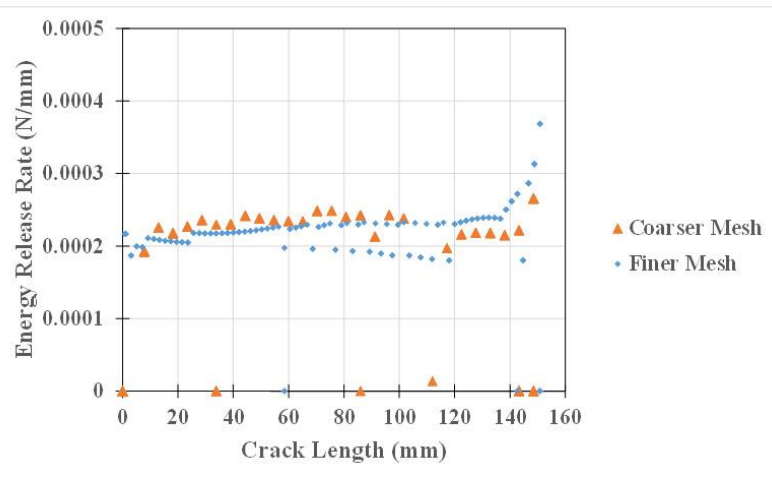

(a)

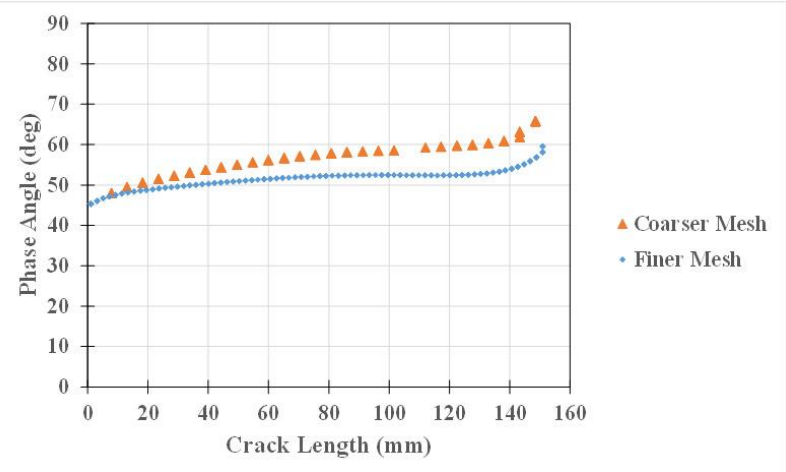

(b) 
Figure 23

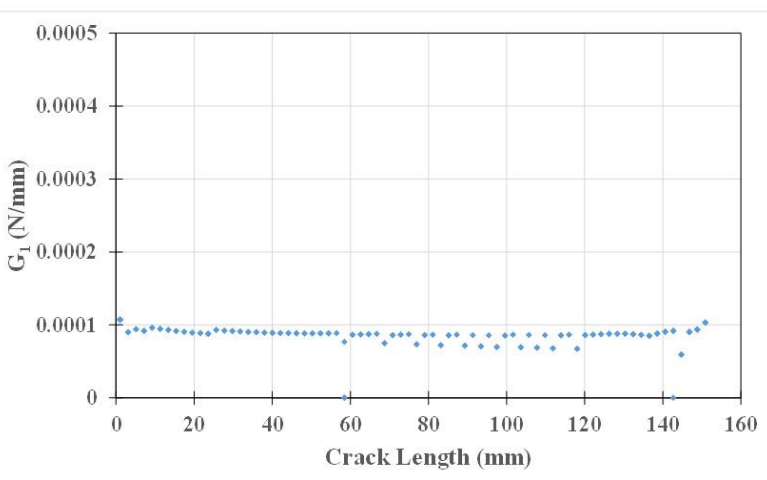

(a)

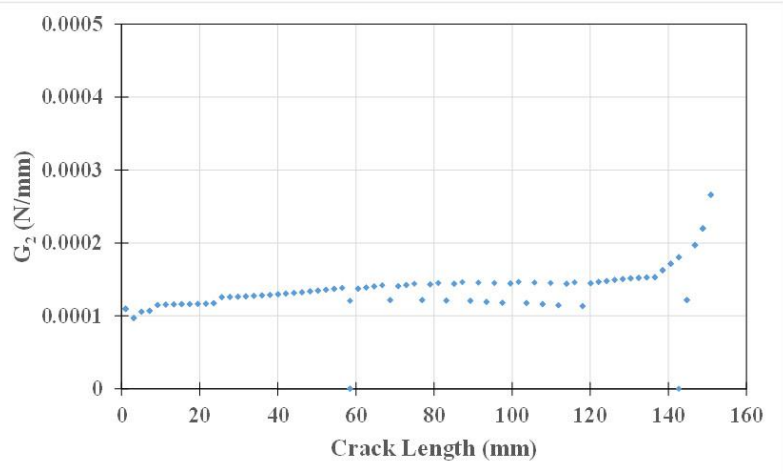

(b) 
Figure 24

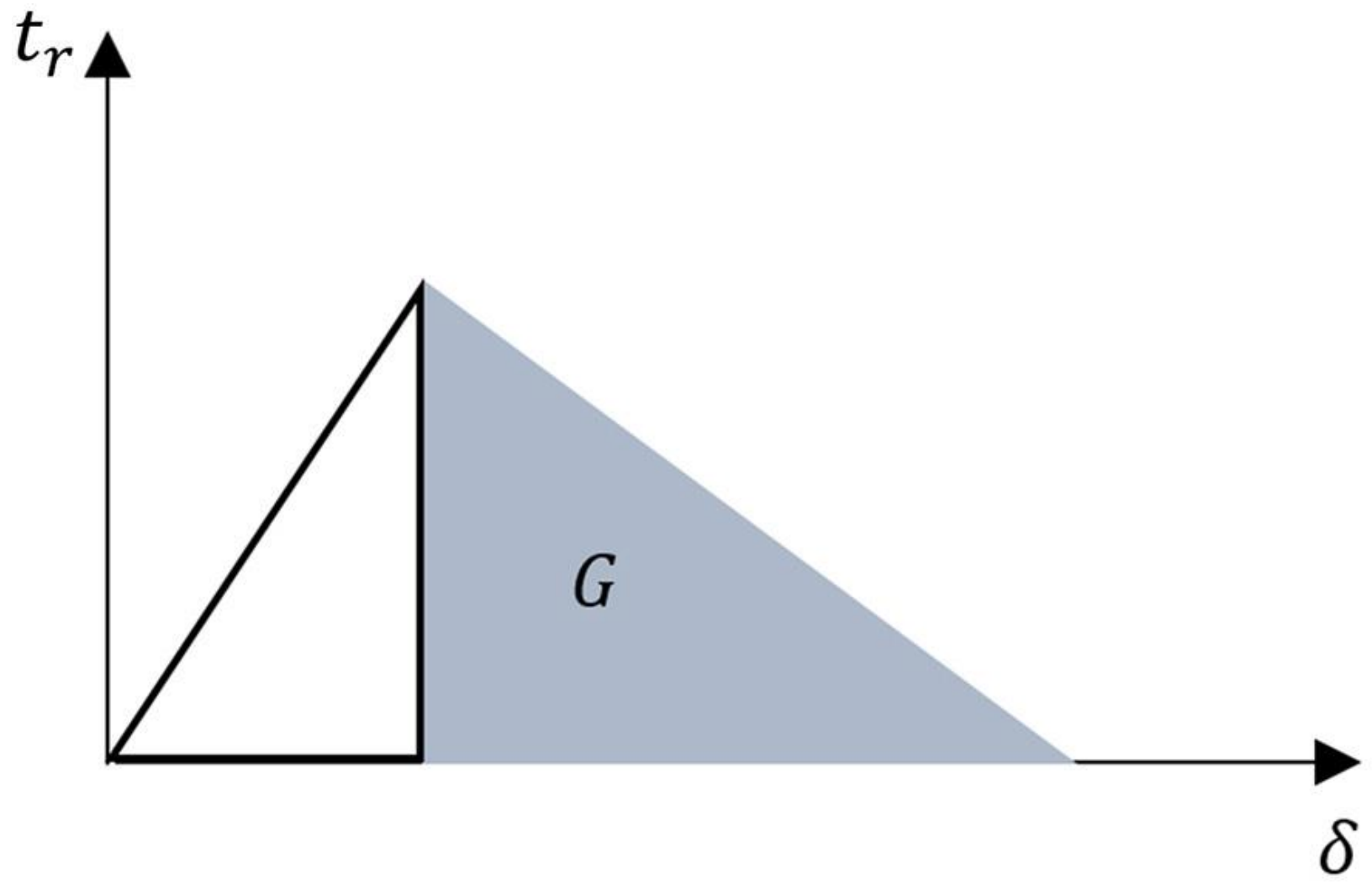


Figure 25

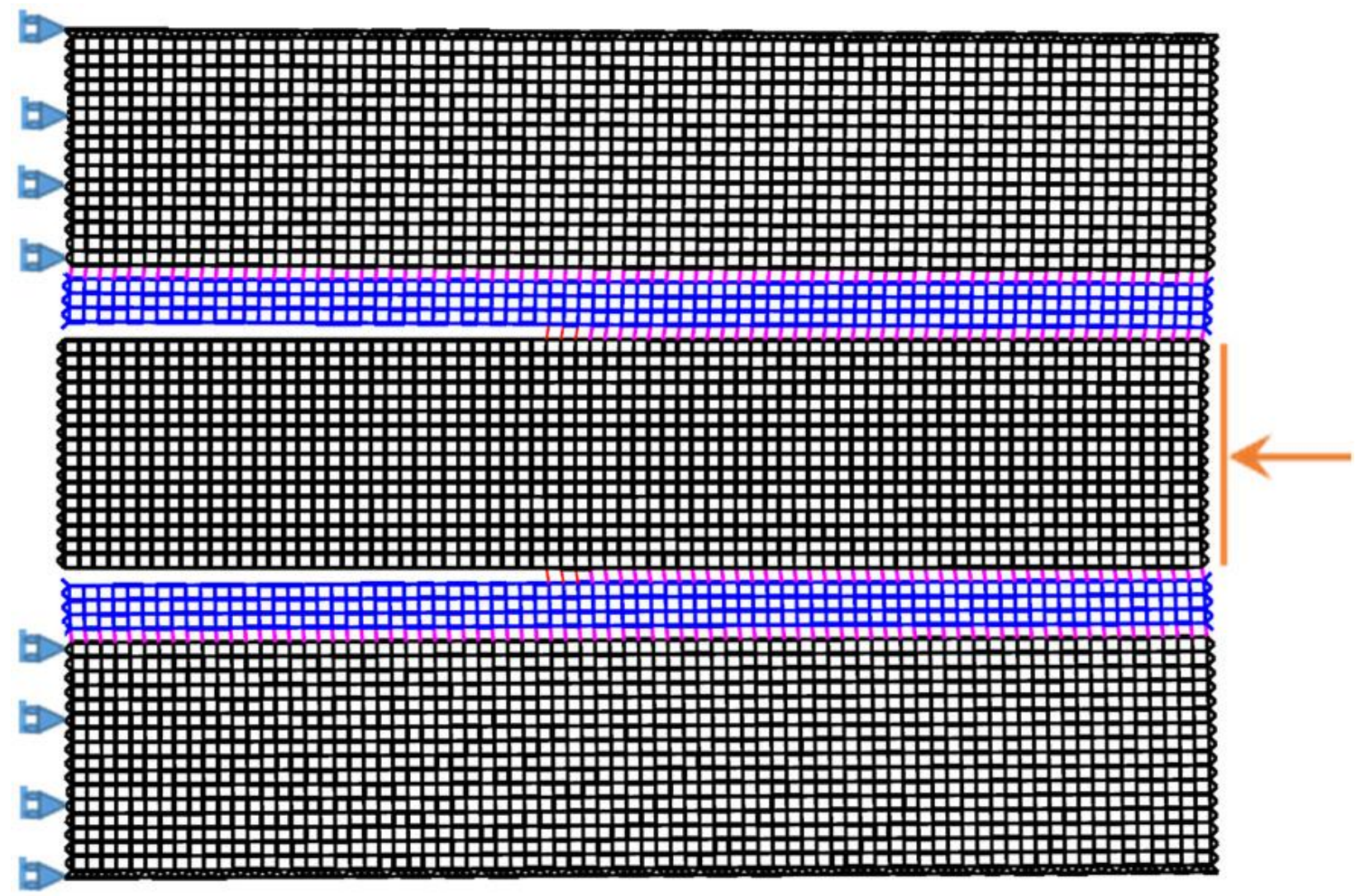


Figure 26

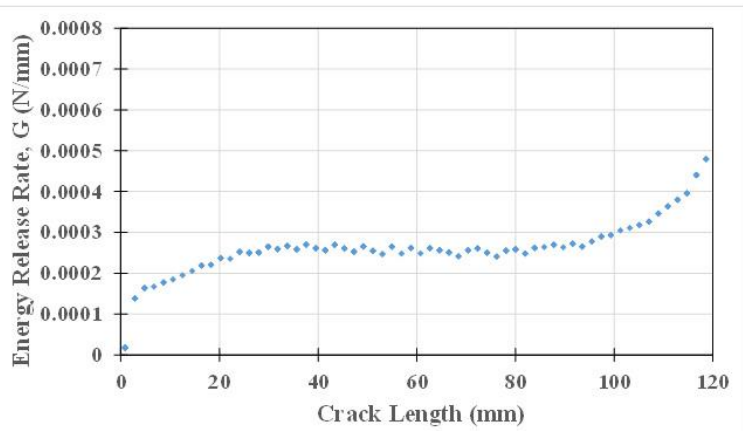

(a)

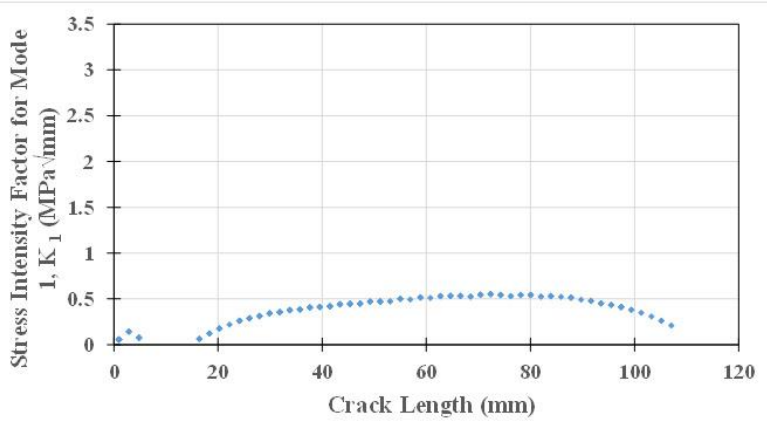

(c)

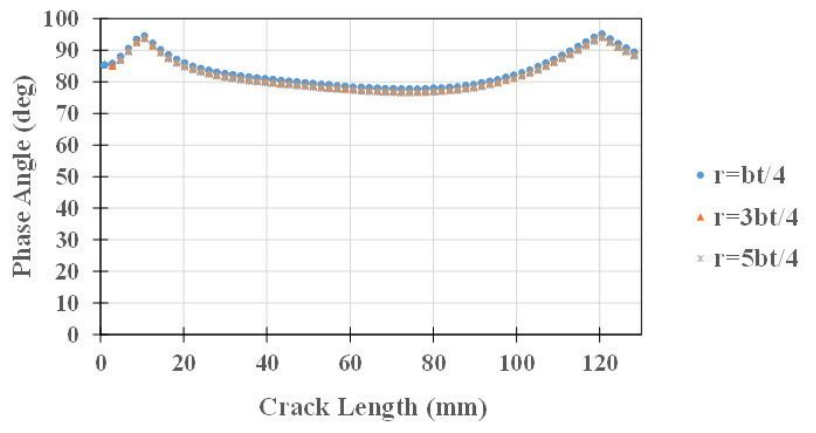

(b)

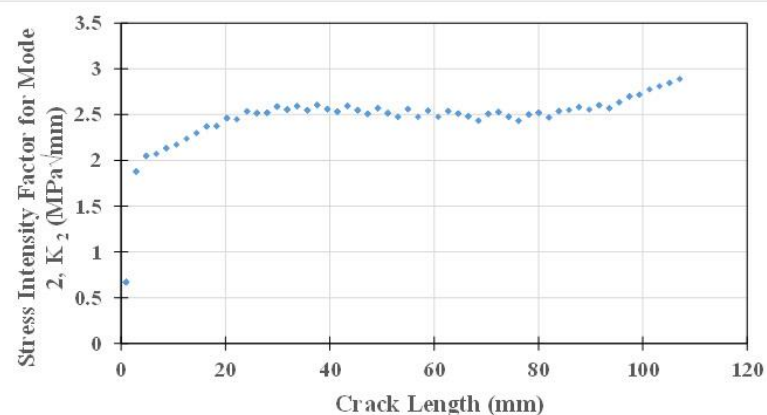

(d) 
Figure 27

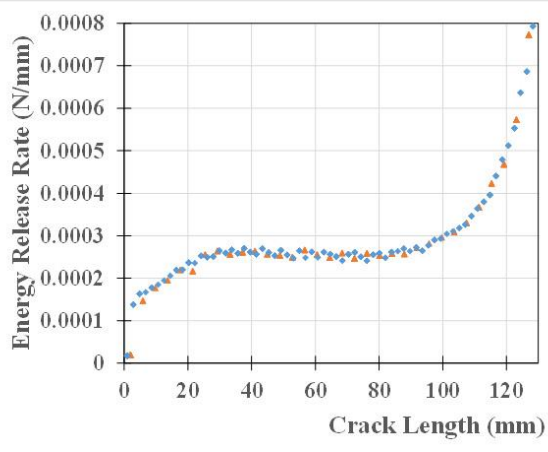

(a)

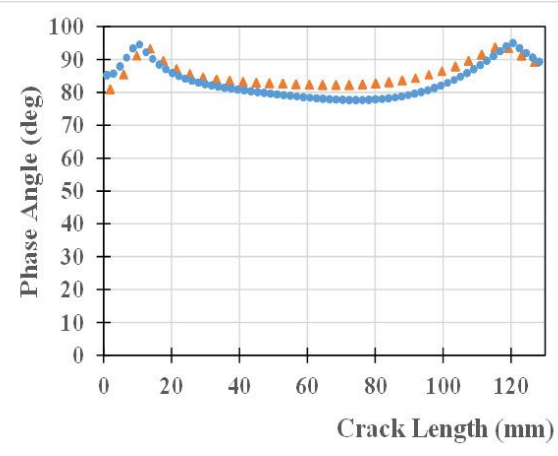

(b)$$
\text { (b) }
$$

^ Coarser Mesh

Finer Mesh 
Figure 28

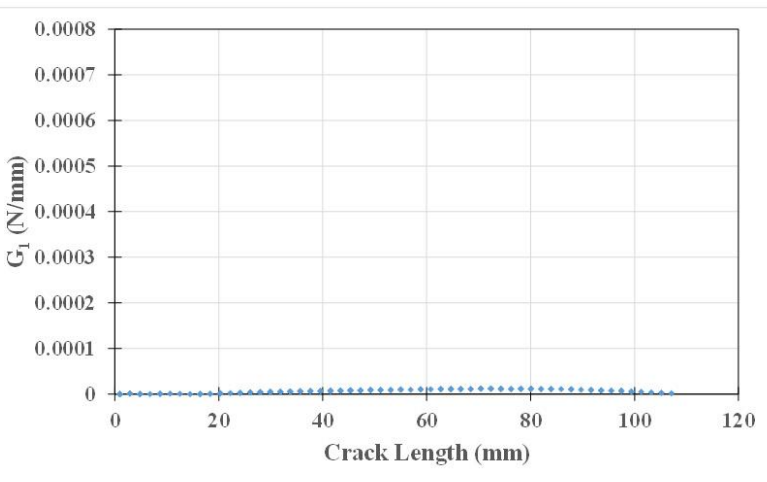

(a)

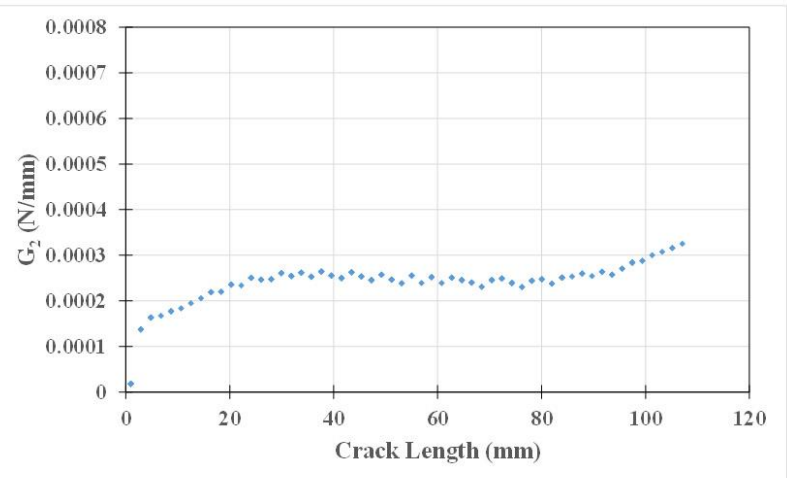

(b) 
Figure 29

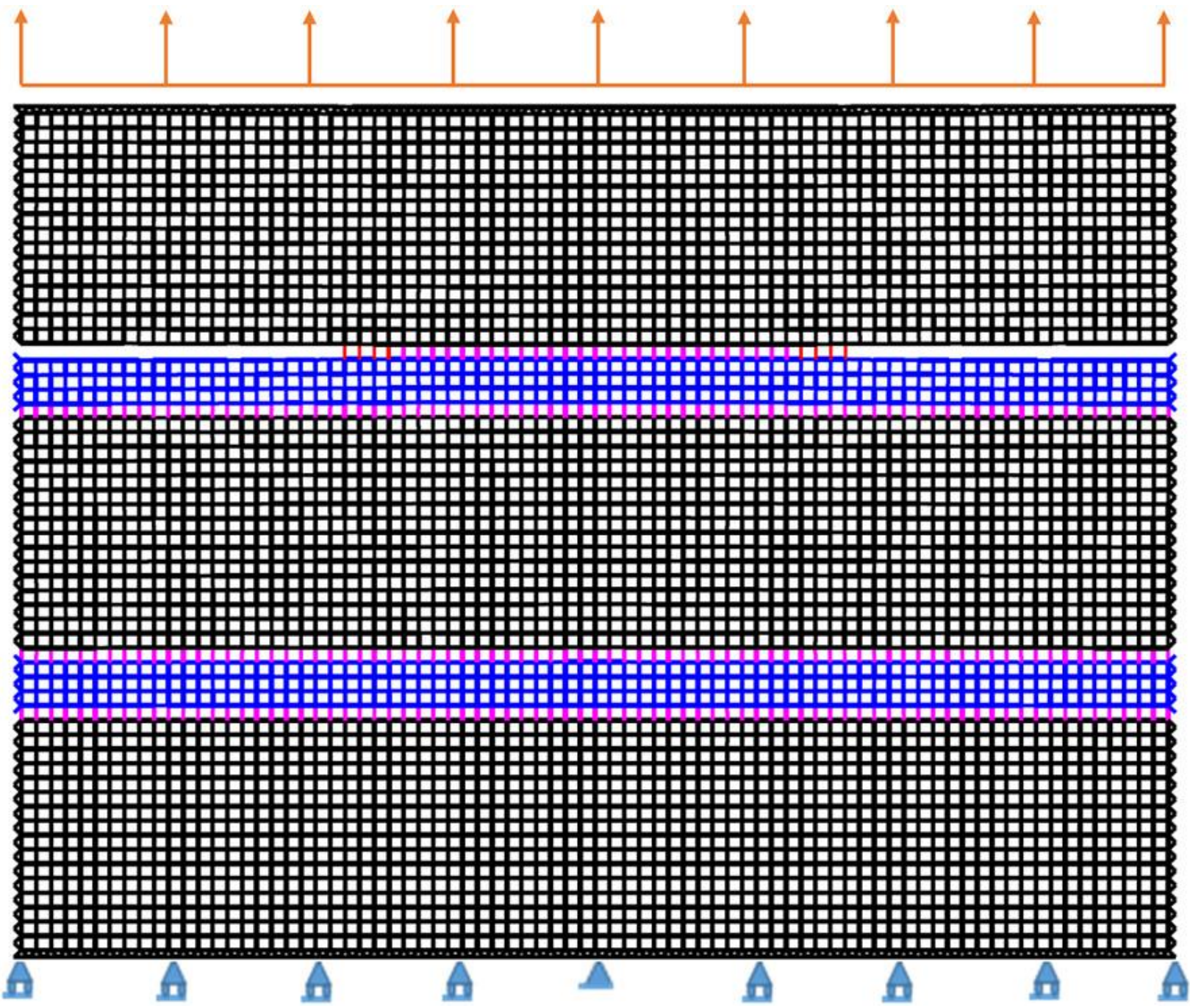


Figure 30

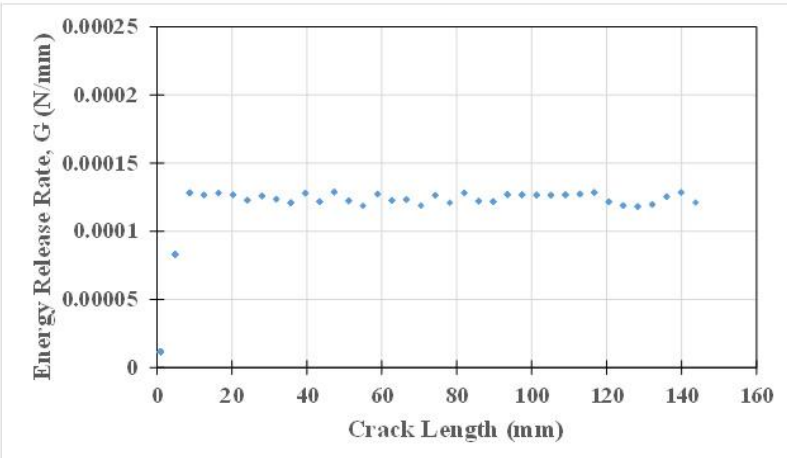

(a)

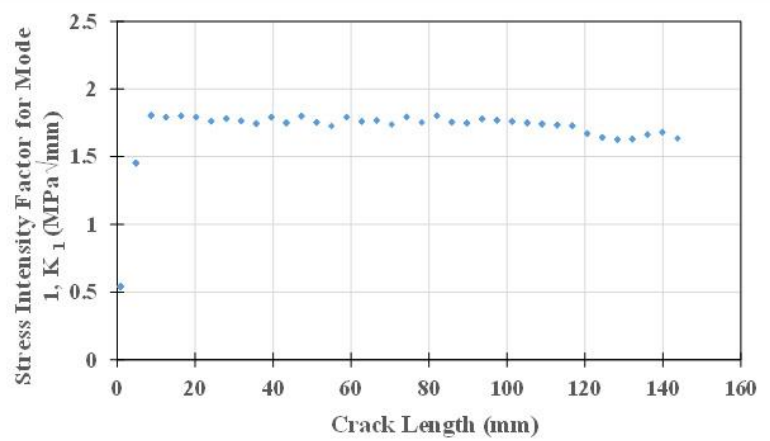

(c)

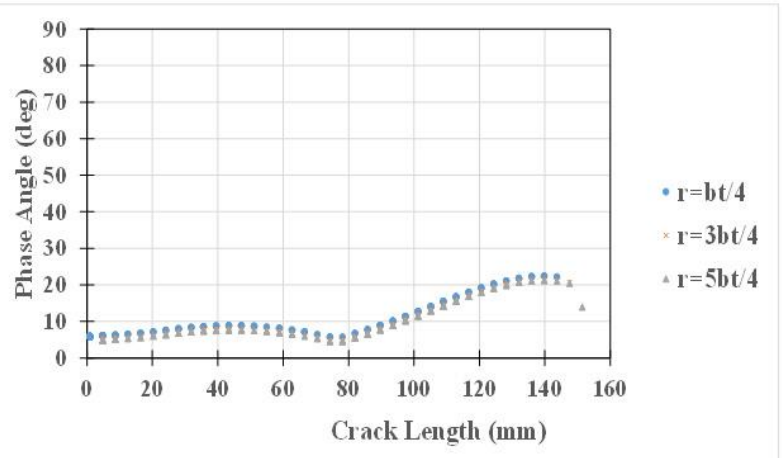

(b)

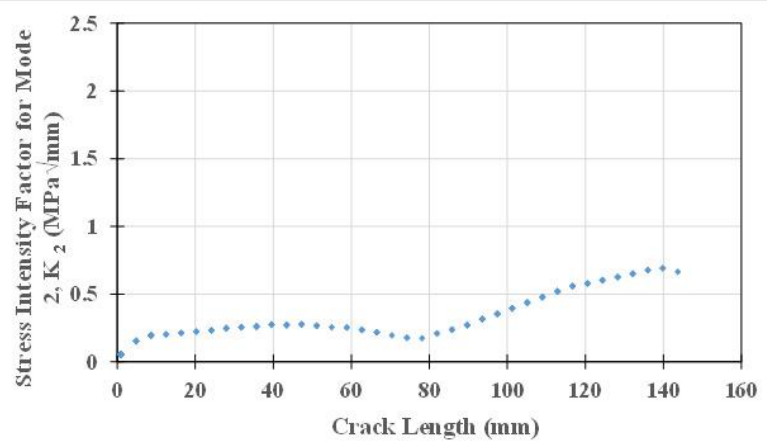

(d) 


\section{Figure 31}

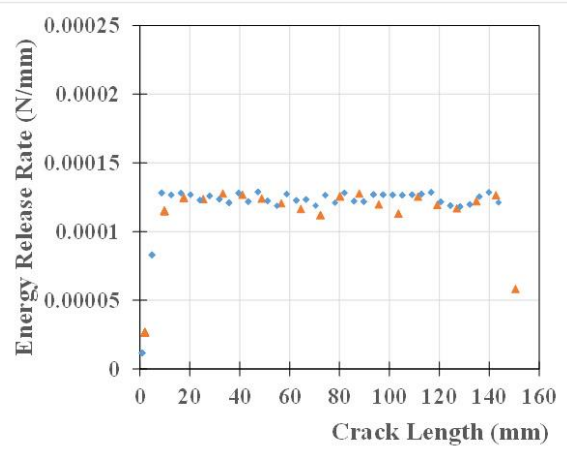

(a) $\triangle$ Coarser Mesh

- Finer Mesh

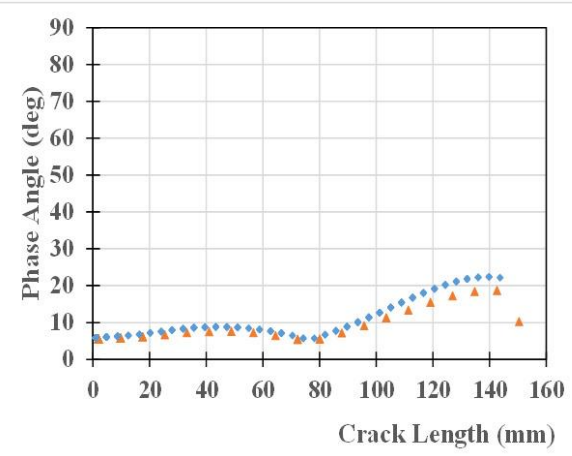

(b) a Coarser Mesh

- Finer Mesh 
Figure 32

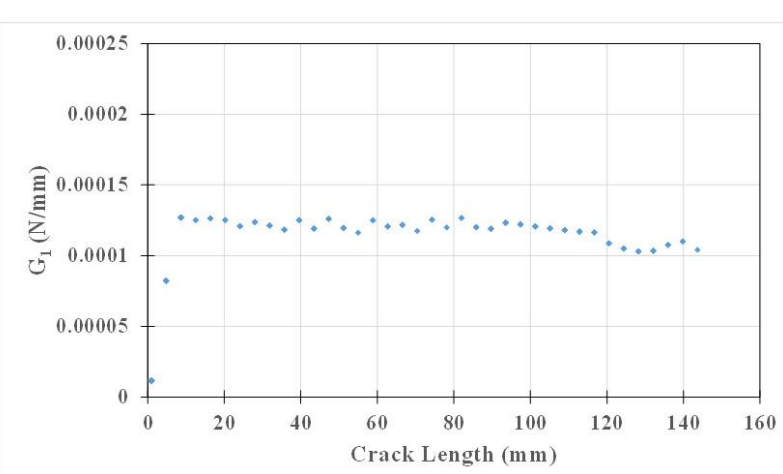

(a)

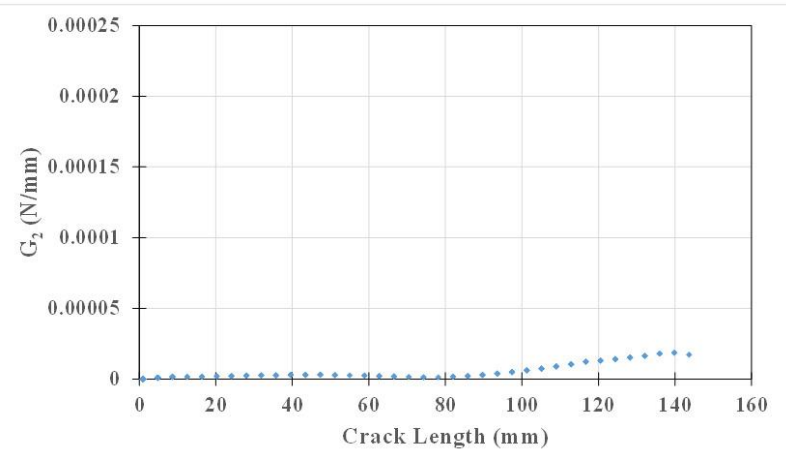

(b) 
Figure 33

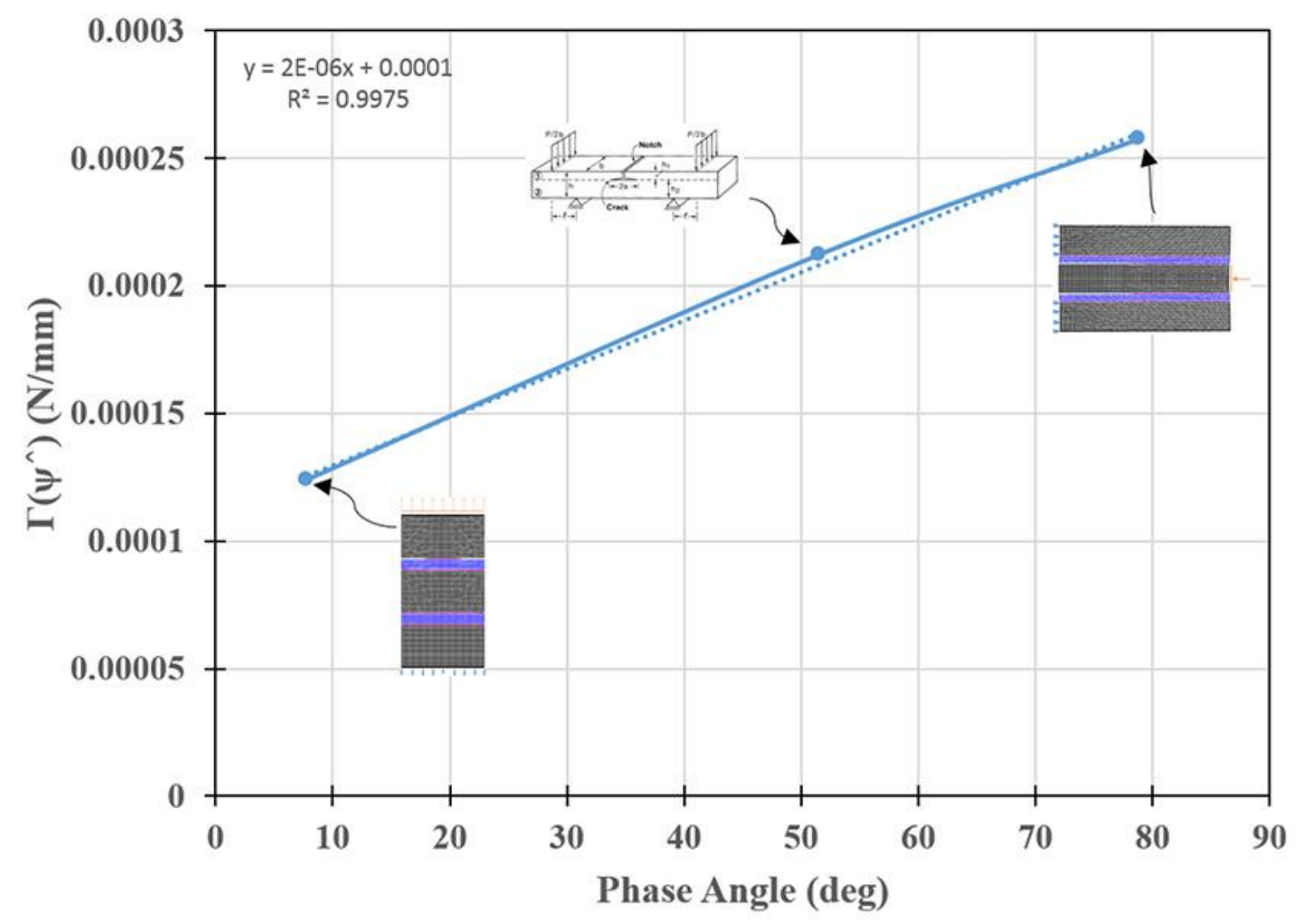


Table 1

Table 1 Mechanical characteristics of materials adopted for the computations

\begin{tabular}{|c|c|c|c|c|c|c|}
\hline & $\begin{array}{c}E\left(l b f / i n^{2}\right) \\
{[M P a]}\end{array}$ & $v$ & $\begin{array}{c}f_{t}\left(l b f / i n^{2}\right) \\
{[M P a]}\end{array}$ & $\begin{array}{c}f_{c}\left(l b f / i n^{2}\right) \\
{[M P a]}\end{array}$ & & \\
\hline Brick & $\begin{array}{c}4506000 \\
{[31067.78]}\end{array}$ & 0.1 & $\begin{array}{c}400 \\
{[2.76]}\end{array}$ & $\begin{array}{c}6000 \\
{[41.37]}\end{array}$ & & \\
\hline \multirow[t]{3}{*}{ Mortar } & $\begin{array}{c}3022000 \\
{[20835.96]}\end{array}$ & 0.2 & $\begin{array}{c}300 \\
{[2.07]}\end{array}$ & $\begin{array}{c}2000 \\
{[13.79]}\end{array}$ & & \\
\hline & $K_{n}\left(l b f / i n^{3}\right)$ & $K_{t}\left(l b f / i n^{3}\right)$ & $f_{t}\left(l b f / i n^{2}\right)$ & $f_{c}\left(l b f / i n^{2}\right)$ & $f_{s}\left(l b f / i n^{2}\right)$ & $\mu_{0}$ \\
\hline & {$\left[\mathrm{N} / \mathrm{mm}^{3}\right]$} & {$\left[\mathrm{N} / \mathrm{mm}^{3}\right]$} & [MPa] & [MPa] & {$[\mathrm{MPa}]$} & \\
\hline Interface & $\begin{array}{l}1504240 \\
{[408.32]}\end{array}$ & $\begin{array}{c}668618 \\
{[181.49]}\end{array}$ & $\begin{array}{c}47 \\
{[0.324]}\end{array}$ & $\begin{array}{c}600 \\
{[4.14]}\end{array}$ & $\begin{array}{c}180 \\
{[1.24]}\end{array}$ & 0.662 \\
\hline
\end{tabular}

